Article

\title{
Synthesis of New 1,3,4-Thiadiazole and 1,2,3,4-Oxathiadiazole Derivatives from Carbohydrate Precursors and Study of Their Effect on Tyrosinase Enzyme
}

\author{
Mohamed M. El-Sadek ${ }^{1}{ }^{*}$, Seham Y. Hassan ${ }^{1}$, Huda E. Abdelwahab ${ }^{1}$ and Galila A. Yacout ${ }^{2}$ \\ 1 Chemistry Department, Faculty of Science, Alexandria University, Alexandria 21231, Egypt; \\ E-Mails: sehamyassen@yahoo.com (S.Y.H.); huda_eid@yahoo.com (H.E.A.) \\ 2 Biochemistry Department, Faculty of Science, Alexandria University, Alexandria 21231, Egypt; \\ E-Mail: galila_69@yahoo.com
}

* Author to whom correspondence should be addressed; E-Mail: elsadek_mm@yahoo.com; Tel.: +20-01-006-544-617; Fax: +20-3-5932-488.

Received: 1 May 2012; in revised form: 10 July 2012 / Accepted: 10 July 2012 /

Published: 11 July 2012

\begin{abstract}
Tetrahydroxybutyl)-2-methylfuran-3-carbohydrazide (2) was condensed with a variety of ketones to afford carbohydrazide derivatives 3-6. Acetylation of 3-5 afforded the acetyl derivatives 7-9, while periodate oxidation of 3-6 afforded the formyl derivatives 10-13. Acid catalyzed condensation of thiosemicarbazide or $o$-tolylthiosemicarbazide with the prepared aldehydes 10-12 gave thiosemicarbazone derivatives 14-19. Cyclization of the latter with acetic anhydride afforded 4,5-dihydro1,3,4-thiadiazolyl derivatives 20-25. On the other hand, condensation of $p$-tosylhydrazine with the prepared aldehydes 10-12 afforded $p$-tosylhydrazone derivatives 26-28. Cyclization of 26-28 with acetic anhydride afforded 1,2,3,4-oxathiadiazole derivatives 29-31 respectively. Moreover, the obtained results regarding to the effect of some of the prepared compounds on tyrosinase enzyme showed that the majority of these compounds having an inhibitory effect; especially compounds $12,16,17$, and 28.
\end{abstract}

Keywords: carbohydrazide; thiosemicarbazone; thiadiazole; oxathiadiazole; tyrosinase 


\section{Introduction}

It was shown that substituted 1,3,4-thiadiazoles exhibit antimicrobial [1] and antitubercular [2-4] activities, while other compounds act on the CNS as anticonvulsants [5-7] or as antidepressant and anxiolitic [8] agents. A family of selective 1,3,4-thiadiazole phosphodiesterase inhibitors [9], and selective orally active cyclooxygenase-2 inhibitors [10] were reported. Moreover, many reports indicate that acylthiosemicarbazides and their corresponding cyclized 1,3,4-thiadiazole derivatives possess anti-inflammatory [11-13] and analgesic [14] activities. 1,3,4-Thiadiazoles are thus a group of heterocycles whose derivatives are important in industry, medicine and agriculture [13,15-21]. Accordingly, in continuation of our work in this area [22-27], a variety of heterocyclic derivatives have been prepared from saccharide derivatives, involving some new thiadiazoles, oxathiadiazoles, and their chemistry and effect of the derivatives on the enzyme tyrosinase was studied [28-31], which is the rate limiting step in melanin biosynthesis [32]. In humans, the main role of the melanins is photoprotection of the skin by absorbing UV radiation that causes DNA damage and the formation of reactive oxygen species (ROS). Human deficiency in melanin causes serious disorders like oculocutaneous albinism and vitiligo. There has also been great interest in the involvement of melanins in malignant melanosomes, the carcinogenic tumors of the skin. Melanoma is most commonly found on the skin, but around $10 \%$ arise in the eyes [33].

In addition, tyrosinase is involved in dopamine biosynthesis, which has been shown to be involved in the control of movements, and the signaling of errors in the prediction of reward, motivation, and cognition. Cerebral dopamine depletion is the hallmark of Parkinson's disease [31]. Other pathological states have also been associated with dopamine dysfunction, such as schizophrenia, autism, and attention deficit hyperactivity disorder [32].

\section{Results and Discussion}

\subsection{Chemistry}

Ethyl 5-(1,2,3,4-tetrahydroxybutyl)-2-methylfuran-3-carboxylate (1) [34] was prepared, then boiled with hydrazine hydrate to give carbohydrazide 2 [35], which when condensed with a variety of ketones afforded carbohydrazides 3-6 in 64-96\% yield (Scheme 1).

The structure of hydrazones 3-6 was proven by their ${ }^{1} \mathrm{H}-\mathrm{NMR}$ spectra, which showed the NH proton as a singlet at $\delta 9.87-9.20$, the proton at position-4 in the furan ring as a singlet at 7.27-6.57 ppm, the 1'-OH proton at 5.06-5.05 and the rest of the sugar protons at the 4.58-4.28 range. The methyl protons at position-2 in the furan ring appeared as a singlet at $\delta 2.44-2.21 \mathrm{ppm}$; additionally the disappearance of the two $\mathrm{NH}_{2}$ protons was observed (see Experimental). It was observed that the C-1' hydroxyl proton of compounds 3-6, resonates at lower field $(5.06-5.05 \mathrm{ppm})$ than the rest of the sugar protons. Electronic deshielding by the adjacent base residue undoubtedly is a major factor in causing these signals to appear at low field. Additional deshielding might also arise through the formation of an intramolecular hydrogen bond with the oxygen of the furan ring. Hydrogen bonding of this type was suggested in polyhydroxyalkyltriazole analogs [36] and polyhydroxyalkylpyrazolo-[3,4-b]-quinoxalines [37] having the D-arabino configuration of the side chain. In addition, the mass spectra of compounds 5 and 6, as examples of the series, showed the corresponding molecular ion peaks at $\mathrm{m} / \mathrm{z} 377$ and 342, 
respectively. On the other hand, acetylation of 3-5 afforded the corresponding acetyl derivatives 7-9 in $45-86 \%$ yield (Scheme 1 ).

Scheme 1. Synthesis of 1,3,4-thiadiazole derivatives.<smiles>CCOC(=O)c1cc(C(O)CO)oc1C</smiles><smiles>CC(=O)CC(=O)NNCC(=O)c1cc(C(=O)NN)oc1C</smiles><smiles>[R]C(C)=NNC(=O)c1cc(C(=O)O)oc1C</smiles>

4: $\mathrm{R}=4-\mathrm{OHC}_{6} \mathrm{H}_{4}$

5: $\mathrm{R}=4-\mathrm{NH}_{2} \mathrm{C}_{6} \mathrm{H}_{4}$

6: $\mathrm{R}=\mathrm{CH}_{2} \mathrm{CH}\left(\mathrm{CH}_{3}\right)_{2}$

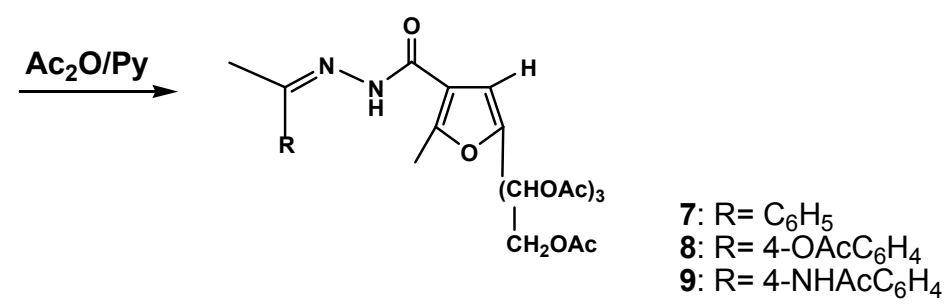
$\mathrm{NaIO}_{4}$<smiles>[R]C(C)=NNC(=O)c1cc(C=O)oc1C</smiles><smiles>[R]N[C@@H]([NH])N</smiles>

10: $\mathrm{R}=\mathrm{C}_{6} \mathrm{H}_{5}$

$\mathrm{NH}_{2} \mathrm{NHCNHR}^{\prime}$

12: $\mathrm{R}=4-\mathrm{NH}_{2}$

13: $\mathrm{R}=\mathrm{CH}_{2} \mathrm{CH}\left(\mathrm{CH}_{3}\right)_{2}$<smiles>[R]NC(=S)N/N=C/c1cc(C(=O)NN=C([R])C)c(C)o1</smiles>

14: $\mathrm{R}=\mathrm{C}_{6} \mathrm{H}_{5}$

$15 \mathrm{R}=4-\mathrm{OHC}_{6} \mathrm{H}_{4}$

16: $\mathrm{R}=4-\mathrm{NH}_{2} \mathrm{C}_{6} \mathrm{H}_{4}$

$\mathrm{R}^{\prime}=\mathrm{H}$

17: $\mathrm{R}=\mathrm{C}_{6} \mathrm{H}_{5}$

$18 \mathrm{R}=4-\mathrm{OHC}_{6} \mathrm{H}_{4}$

$\mathrm{R}^{\prime}=\mathrm{H}$

19: $\mathrm{R}=4-\mathrm{NH}_{2} \mathrm{C}_{6} \mathrm{H}_{4}$

$\mathrm{R}^{\prime}=2-\mathrm{CH}_{3} \mathrm{C}_{6} \mathrm{H}_{4}$

$\mathrm{R}^{\prime}=2-\mathrm{CH}_{3} \mathrm{C}_{6} \mathrm{H}_{4}$

$\mathrm{R}^{\prime}=2-\mathrm{CH}_{3} \mathrm{C}_{6} \mathrm{H}_{4}$<smiles>[R]C(C)=NN([PH])C(=O)c1cc(C2=NN(C(C)=O)C(N(P)C(C)=O)S2)oc1C</smiles>

20: $\mathrm{R}=\mathrm{C}_{6} \mathrm{H}_{5}$

$21 \mathrm{R}=4-\mathrm{OAcC}_{6} \mathrm{H}_{4}$

22: $\mathrm{R}=4-\mathrm{NHACC}_{6} \mathrm{H}_{4}$

23: $\mathrm{R}=\mathrm{C}_{6} \mathrm{H}_{5}$

24: $\mathrm{R}=4-\mathrm{OAcC}_{6} \mathrm{H}_{4}$

$\mathrm{R}^{\prime}=\mathrm{H}$

$\mathrm{R}^{\prime}=\mathrm{COCH}_{3}$

$\mathrm{R}^{\prime \prime}=\mathrm{H}$

$\mathrm{R}^{\prime}=\mathrm{COCH}_{3}$

$\mathrm{R}^{\prime \prime}=\mathrm{H}$

$\mathrm{R}^{\prime}=2-\mathrm{CH}_{3} \mathrm{C}_{6} \mathrm{H}_{4}$

$\mathrm{R}^{\prime \prime}=\mathrm{H}$

25: $\mathrm{R}=4-\mathrm{N}(\mathrm{Ac})_{2} \mathrm{C}_{6} \mathrm{H}_{4}$

$\mathrm{R} "=\mathrm{COCH}_{3}$

$\mathrm{R}^{\prime \prime}=\mathrm{H}$

$\mathrm{R}^{\prime \prime}=\mathrm{H}$ 
The ${ }^{1} \mathrm{H}-\mathrm{NMR}$ spectra of compound $\mathbf{8}$ and $\mathbf{9}$ showed the disappearance of the $\mathrm{OH}$ protons in the sugar region, the $O$-acetyl protons at $\delta 2.43$ and $2.23 \mathrm{ppm}$, respectively, and peaks at 2.59, 2.03 for the $\mathrm{N}$ - acetyl protons, respectively (for the other protons see the Experimental). Periodate oxidation of compounds 3-6 afforded the corresponding formyl derivatives 10-13, in 36-65\% yields (Scheme 1).

${ }^{1} \mathrm{H}-\mathrm{NMR}$ spectra of compounds 10-12 showed the NH proton as a singlet at $\delta 9.32,8.91$ and $9.36 \mathrm{ppm}$. respectively, and the formyl group proton as a singlet at $\delta 9.87,9.34$, and $9.44 \mathrm{ppm}$, respectively (for other protons see the Experimental). The mass spectrum of compound 13, showed the molecular ion peak at $\mathrm{m} / \mathrm{z} 250$, which was also the base peak.

Acid catalyzed condensation of thiosemicarbazide or $o$-tolylthiosemicarbazide with the prepared formyl derivatives 10-12 gave thiosemicarbazone derivatives 14-19 in 43-99\% yield (Scheme 1). The mass spectra of compounds 14, 16-18 showed the molecular ion peaks at $m / z 343,358,433$ and 449 respectively. Cyclization of the prepared compounds 14-19 with acetic anhydride afforded 1,3,4-thiadiazole derivatives $\mathbf{2 0}-\mathbf{2 5}$ in $40-73 \%$ yield (Scheme 1).

The ${ }^{1} \mathrm{H}-\mathrm{NMR}$ spectra of compounds 20-22 showed the disappearance of the $\mathrm{NH}_{2}$ protons and $\mathrm{CH}=\mathrm{N}$ proton. Instead, the $\mathrm{N}-\mathrm{Ac}$ methyl protons appeared as a singlet. Interestingly, it was noted that the ${ }^{1} \mathrm{H}-\mathrm{NMR}$ spectra of compounds 21 and $\mathbf{2 2}$ showed the proton at position-4 in the furan ring as a doublet signal at $\delta 6.69$ and 7.22 instead of a singlet signal due to the long rang interaction between $\mathrm{H}$-furan and the NH proton of the amide group. However a theoretical study of the NMR of compound 21 was attempted whereby the stable conformer of this compound was first established using the universal force field UFF molecular mechanics method (Table 1). After that the $\{\mathrm{B} 3 \mathrm{LYP} / 6-31 \mathrm{G}(\mathrm{d})\}$ density functional approach was used to fine tune the geometry of the compound. The Orca computational chemistry program was used in this step. According to the calculation the distance between the NH proton and the $\mathrm{H}$-furan is equal to $2.304 \AA$, which is the same value of the distance between the methylene protons and the methyl protons in the ethanol molecule. In the same way, $\mathrm{H}$-furan appeared as a doublet due to the coupling interaction with the $\mathrm{NH}$ proton, while the proton of the NH group appears as a singlet, so the question is why the interaction with the H-furan didn't affect the signal of $(\mathrm{NH})$ proton. This is attributed to the ionization factor [38] (Figures 1 and 2).

Table 1. The proton NMR isotropic shift of compound $\mathbf{2 1}$ calculated theoretically at the level 6-311G $(\mathrm{d}, \mathrm{p})$ using the Orca program and compared with the experimental values.

\begin{tabular}{lll}
\hline Proton & Calculated & Experimental \\
\hline H-1 & 2.42 & 2.24 \\
H-2 & 2.62 & 2.27 \\
H-3 & 7.02 & 8.22 \\
H-4 & 6.62 & 6.69 \\
H-5 & 7.82 & 7.06 \\
H-6 & 2.22 & 2.14 \\
H-7 & 2.39 & 2.34 \\
H-8 & 7.32 & $7.23-7.26$ \\
H-9 & 7.32 & $7.23-7.26$ \\
H-10 & 2.22 & 2.21 \\
H-11 & 2.39 & 2.34 \\
\hline
\end{tabular}


Figure 1. The distance between $\mathrm{NH}$ proton and $\mathrm{H}$-furan is equal to the distance between the methylene protons and the methyl protons in the ethanol molecule.

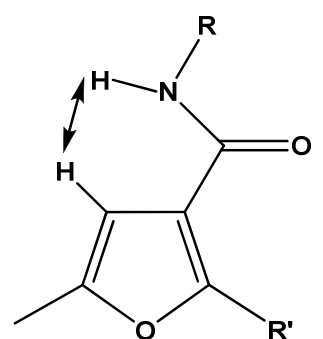

21<smiles>CCCO</smiles>

ethanol

Figure 2. Orca Computational Chemistry program of the compound 21.

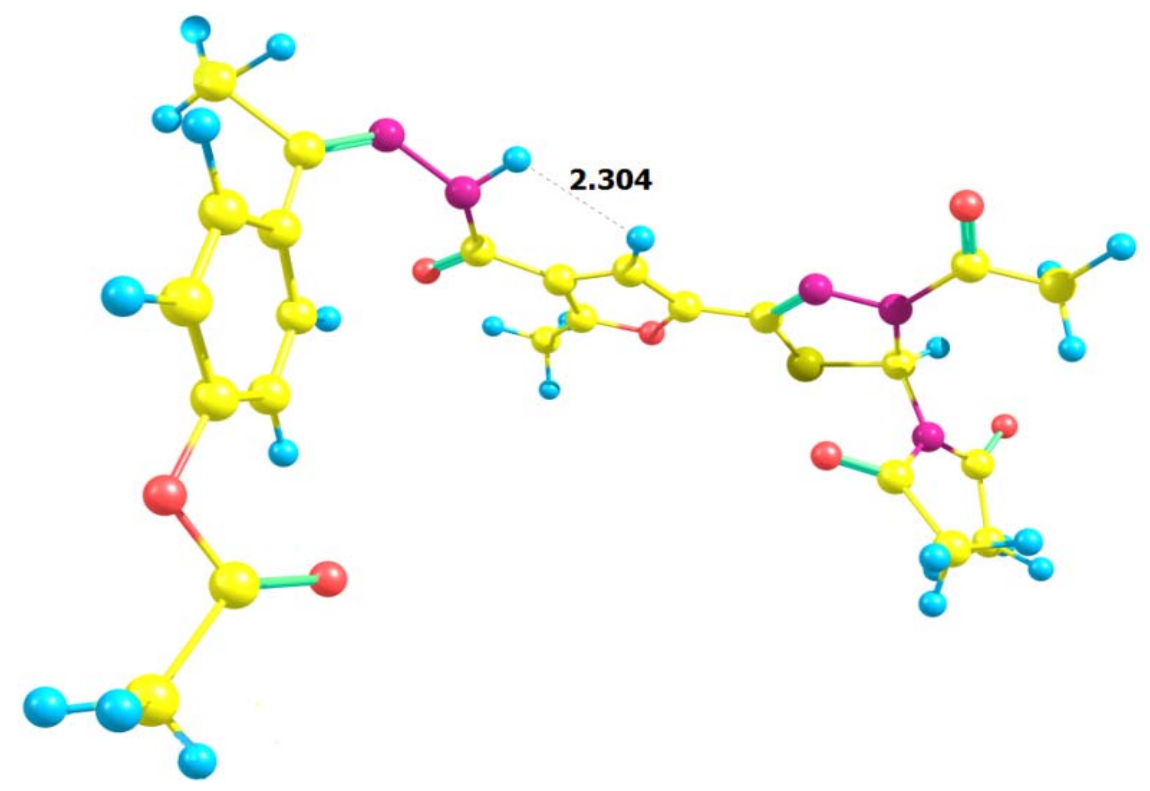

The mass spectra of compounds 20 and 22 showed the molecular ion peaks at $\mathrm{m} / \mathrm{z} 427$ and 526, respectively. The mass spectrum of compound $\mathbf{2 3}$ showed the molecular ion peak at $\mathrm{m} / \mathrm{z} 560$. In addition, condensation of $p$-tosylhydrazine with the formyl derivatives $\mathbf{1 0}-\mathbf{1 2}$ afforded $p$-tosylhydrazone derivatives 26-28 respectively in 42-83\% yield (Scheme 2). The ${ }^{1} \mathrm{H}-\mathrm{NMR}$ spectra of compounds 26 and 27 showed the disappearance of the aldehyde proton. The two NH protons showed as a singlet at $\delta 9.36,9.71$, and 9.36, 10.47, respectively, the $\mathrm{CH}_{3}$ protons of the $p$-tolyl moiety as a singlet at $\delta 2.36$ and $2.32 \mathrm{ppm}$, respectively (see Experimental part). The mass spectra of compounds 26 and 27 showed the molecular ion peaks at $m / z 438$ and 454, respectively.

Similarly, cyclization of these hydrazones 26-28, with acetic anhydride afforded 1,2,3,4oxathiadiazole derivatives 29-31 in 32-54\% yield (Scheme 2). The ${ }^{1} \mathrm{H}-\mathrm{NMR}$ spectra of the compounds 29 and 31 showed the disappearance of both the $\mathrm{CH}=\mathrm{N}$ and the $\mathrm{NHSO}_{2}$ proton signals. The ${ }^{1} \mathrm{H}-\mathrm{NMR}$ spectra showed the $\mathrm{CH}_{3}$ protons of the $p$-tolyl group as a singlet at $\delta 2.40,2.49 \mathrm{ppm}$, the $\mathrm{CH}_{3}-\mathrm{C}=\mathrm{N}$ protons as a singlet at $\delta 2.05,2.09$ and the $\mathrm{CH}_{3}$-furan protons as a singlet at $\delta 2.40,2.49$, respectively. The mass spectra of compounds 29 and 30 showed the molecular ion peaks at $\mathrm{m} / \mathrm{z} 420$ and 478 , respectively. 
Scheme 2. Synthesis of oxathiadiazole derivatives.
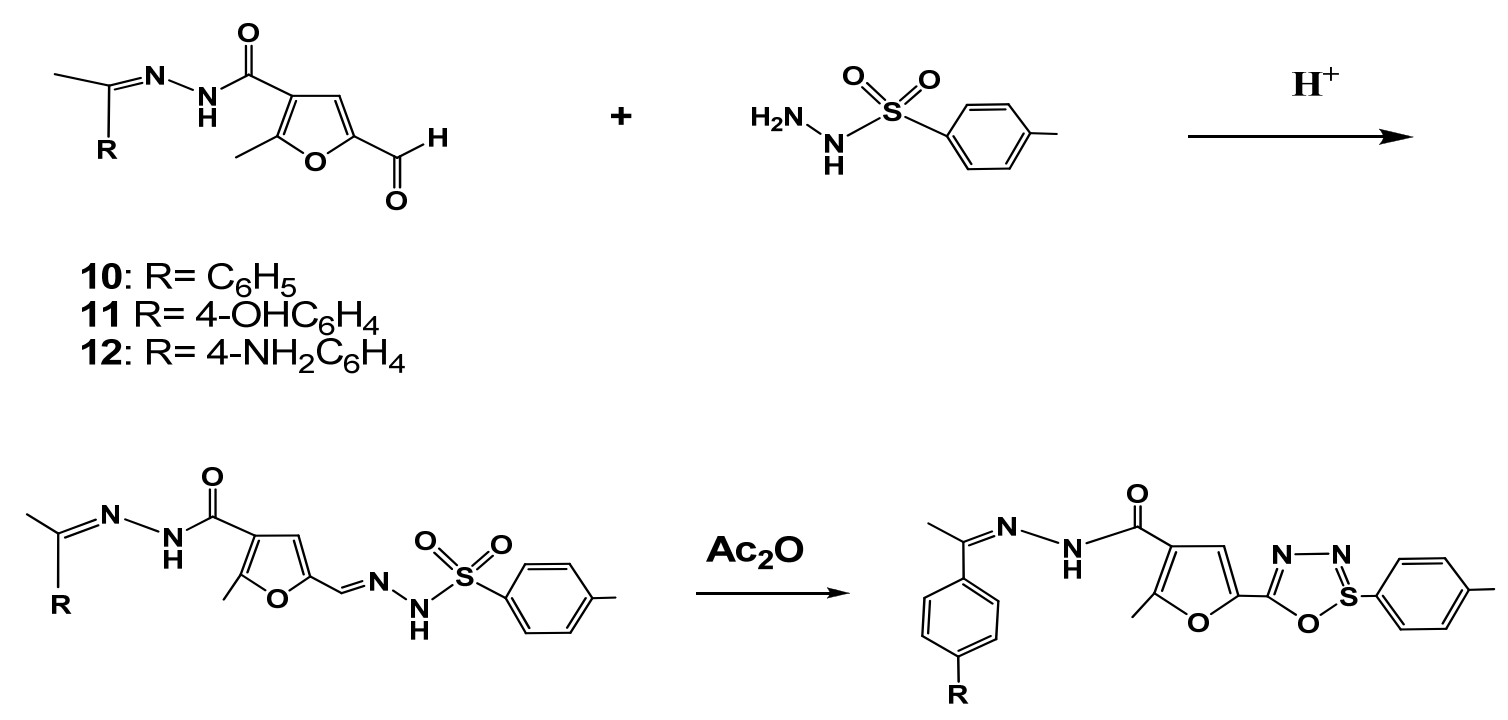

26: $\mathrm{R}=\mathrm{C}_{6} \mathrm{H}_{5}$

27: $\mathrm{R}=4-\mathrm{OHC}_{6} \mathrm{H}_{4}$

28: $\mathrm{R}=4-\mathrm{NH}_{2} \mathrm{C}_{6} \mathrm{H}_{4}$

29: $\mathrm{R}=\mathrm{C}_{6} \mathrm{H}_{5}$

30: $\mathrm{R}=4-\mathrm{OAcC}_{6} \mathrm{H}_{4}$

31: $\mathrm{R}=4-\mathrm{NHACC}_{6} \mathrm{H}_{4}$

\subsection{Biological Activity Assay}

Tyrosinase was prepared from mushrooms in a phosphate buffer $(50 \mathrm{mM}, \mathrm{pH} 6.0)$ according to the method of Yang and Robb [39], and the obtained supernatant after centrifugation was used as a source of enzyme.

\subsubsection{Enzyme Activity Assay}

The activity of the prepared enzyme solution was determined by following the formation of dopachrome spectrophotometrically at $30{ }^{\circ} \mathrm{C}$, after addition of $50 \mu \mathrm{L}$ enzyme preparation to a cuvette containing $1.2 \mathrm{~mL}$ phosphate buffer $(50 \mathrm{mM}, \mathrm{pH} 6.0)$ and $0.8 \mathrm{~mL}$ L-Dopa $(10 \mathrm{mM})$, the solution was immediately mixed and the increase in absorbance at $475 \mathrm{~nm}$ (indicating the formation of dopachrome) was recorded using UV-20100-spectrophotometer. Blank experiment was carried out as mentioned above using $50 \mu \mathrm{L}$ of buffer instead of enzyme preparation [40].

\subsubsection{Enzyme Activity Assay in Presence of Compounds 10-12, 14-19, 26-28}

The effect of the presence of compounds 10-12, 14-19, 26-28 on tyrosinase activity, was determined separately by following the above steps for dopachrome formation then recording the increase in absorbance at $475 \mathrm{~nm}$ at time intervals (0-180 s), as shown in Table 2, and Figures 3 and 4. All tests were carried out in duplicate. 
Table 2. Effect of time on the velocity of tyrosinase-catalyzed reaction in presence of carbohydrazide derivatives (10-12), (14-19), and (26-28) compared to control enzyme.

\begin{tabular}{|c|c|c|c|c|c|c|c|c|c|c|c|c|c|}
\hline \multirow{2}{*}{ Time (s) } & \multicolumn{13}{|c|}{ Rate (v) } \\
\hline & Control enzyme & 10 & 11 & 12 & 14 & 15 & 16 & 17 & 18 & 19 & 26 & 27 & 28 \\
\hline $\mathbf{0}$ & 0.118 & 0.325 & 0.0605 & 0.107 & 0.148 & 0.077 & 0.05 & 0.069 & 0.98 & 0.237 & 1.897 & 0.092 & 0.0245 \\
\hline 30 & 0.309 & 0.31 & 0.1235 & 0.115 & 0.132 & 0.078 & 0.052 & 0.182 & 1.01 & 0.402 & 1.982 & 0.082 & 0.0455 \\
\hline 60 & 0.502 & 0.243 & 0.1725 & 0.0825 & 0.119 & 0.083 & 0.054 & 0.329 & 1.25 & 0.625 & 2.056 & 0.063 & 0.0465 \\
\hline 90 & 0.702 & 0.145 & 0.2225 & 0.073 & 0.06 & 0.085 & 0.055 & 0.55 & 1.49 & 0.818 & 2.109 & 0.074 & 0.0565 \\
\hline 120 & 0.893 & 0.118 & 0.266 & 0.0725 & 0.046 & 0.088 & 0.057 & 0.71 & 2.07 & 0.963 & 2.177 & 0.089 & 0.064 \\
\hline 150 & 1.063 & 0.131 & 0.3015 & 0.082 & 0.043 & 0.091 & 0.059 & 0.92 & 2.3 & 1.385 & 2.26 & 0.086 & 0.0735 \\
\hline 180 & 1.192 & 0.151 & 0.3335 & 0.0805 & 0.045 & 0.094 & 0.06 & 1.08 & 2.58 & 1.68 & 2.31 & 0.06 & 0.082 \\
\hline
\end{tabular}

Figure 3. Effect of time on the rate of tyrosinase-catalyzed reaction in presence of compounds 18, 19, and 26 compared to control enzyme.

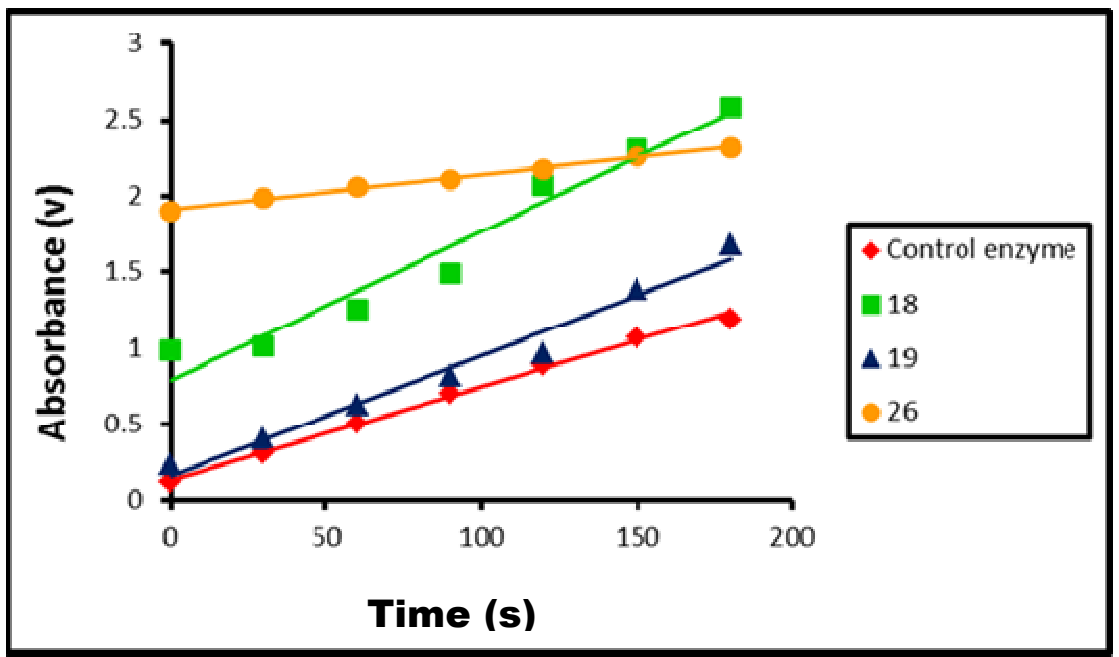

\subsubsection{Results}

The obtained results showed that all these compounds are inhibitors for tyrosinase, except for compounds 18, 19 and $\mathbf{2 6}$ which were found to be activators of tyrosinase.

\subsubsection{Type of Inhibition}

The type of inhibition of $N^{\prime}$-(1-(4-aminophenyl)ethylidene)-5-formyl-2-methylfuran-3-carbohydrazide (12), $N^{\prime}$-(1-(4-aminophenyl)ethylidene)-5-formyl-2-methylfuran-3-carbohydrazide (16) and 1-((4-(1-(4-aminophenylethylideneaminocarbamoyl)furan-2-yl)methylene-2-tosylhydrazine (28) on enzyme activity was detected by plotting $1 /[\mathrm{S}]$ against 1/v using different concentrations of dopa (3, 6, $10,15,20 \mathrm{mM}$ ) according to the abovementioned steps. Compound $\mathbf{1 2}$ showed a highly competitive inhibition, with $\mathrm{V}_{\max }$ (maximum rate, 0.33) and $\mathrm{K}_{\mathrm{m}}$ (Michaelis constant, 8.24), while both compound 16 and compound 28 showed an uncompetitive inhibition with $\mathrm{V}_{\max }(0.0667)$ and $\mathrm{K}_{\mathrm{m}}(0.763)$, and $\mathrm{V}_{\max }$ (0.074) and $K_{m}(0.444)$, respectively. 
Figure 4. Effect of time on the rate of tyrosinase-catalyzed reaction in presence of compounds 10-12, 14-17, 27 and 28 compared to control enzyme.

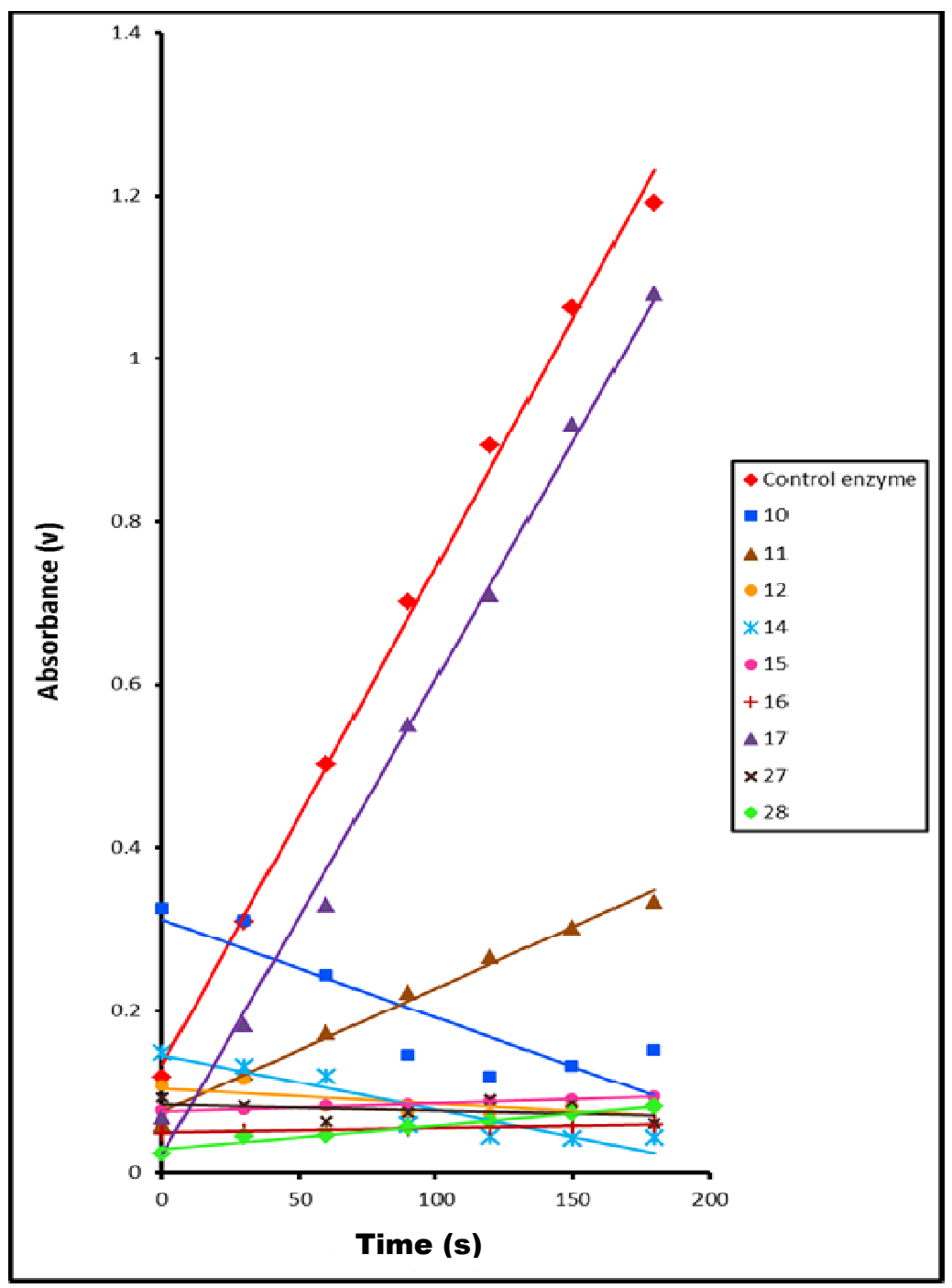

\section{Experimental}

\subsection{General Methods}

Melting points were determined on a Koffler block and are uncorrected. IR spectra were recorded on Perkin Elmer 1600 USA Spectrometer. ${ }^{1} \mathrm{H}-\mathrm{NMR}$ were recorded on a JEOL JNM ECA $500 \mathrm{MHz}$ instrument using tetramethylsilane as an internal standard. Mass spectra were recorded on a GC-MS solution DI Analysis Shimadzu Qp-2010 instrument. Elemental analysis was determined at the Regional Center for Mycology and Biotechnology, Al-Azhar University Plus. Optical rotation was obtained at $22{ }^{\circ} \mathrm{C}$ with a Perkin-Elmer model 241 Polarimeter equipped with a $10 \mathrm{~cm}, 1 \mathrm{~mL}$ micro cell. Thin layer chromatography (TLC) was carried out on silica gel plates. Solutions were evaporated under diminished pressure unless otherwise stated. The ChemDraw-Ultra-8.0 software has been used to name the prepared compounds. 


\subsection{Reactions of Carbohydrazide $\mathbf{2}$ with Ketones}

A solution of 5-(1,2,3,4-tetrahydroxybutyl)-2-methylfuran-3-carbohydrazide 2 (2.5 g, $0.01 \mathrm{~mol})$ [31,32] in ethanol $(50 \mathrm{~mL})$ containing $\mathrm{AcOH}(0.1 \mathrm{~mL})$ was treated with ketone $(0.01 \mathrm{~mol})$. The mixture was refluxed for $8 \mathrm{~h}$. After cooling, the product that separated out was filtered off, washed with a little ethanol and dried.

5-(1,2,3,4-Tetrahydroxybutyl)-2-methyl-N-(1-phenylethylidene)furan-3-carbohydrazide (3). Yield 96.4\%. Rwcrystallized from ethanol as canary yellow crystals; m.p. $144-145{ }^{\circ} \mathrm{C} ; \mathrm{R}_{\mathrm{f}}: 0.97$ $\left(\mathrm{CHCl}_{3} / \mathrm{MeOH}, 20: 1, \mathrm{v} / \mathrm{v}\right) ;[\alpha]_{\mathrm{D}}{ }^{20}-19.2$; IR $(\mathrm{KBr}): 1564(\mathrm{C}=\mathrm{N}), 1642(\mathrm{CONH}), 3055(\mathrm{NH}), 3340 \mathrm{~cm}^{-1}$ $(\mathrm{OH}) ;{ }^{1} \mathrm{H}-\mathrm{NMR}$ (DMSO-d $\left.d_{6}\right) \delta: 1.21$ (s, 3H, $\mathrm{CH}_{3} \mathrm{CN}$ ), 2.34 (s, 3H, $\mathrm{CH}_{3}$-furan), 3.33-3.38 (m, 1H, H-3'), 3.41-3.44 (m, 1H, H-2'), 3.47-3.53 (m, 2H, H-4a', H-4b'), 4.28-4.33 (m, 1H, 4'-OH; exchangeable with $\left.\mathrm{D}_{2} \mathrm{O}\right), 4.41\left(\mathrm{~d}, 1 \mathrm{H}, 3^{\prime}-\mathrm{OH} ; J=7.7 \mathrm{~Hz}\right.$, exchangeable with $\left.\mathrm{D}_{2} \mathrm{O}\right), 4.57$ (d, 1H, 2'-OH; $J=5.4 \mathrm{~Hz}$, exchangeable with $\left.\mathrm{D}_{2} \mathrm{O}\right), 4.69$ (d, 1H, H-1'; $\left.J=4.6 \mathrm{~Hz}\right), 5.06$ (d, 1H, 1'-OH; $J=5.4 \mathrm{~Hz}$, exchangeable with $\mathrm{D}_{2} \mathrm{O}$ ), 7.27 (s, 1H, H-furan), 7.42-7.44 (m, 3H, Ar-H), 7.91-7.93 (m, 2H, Ar-H), 9.87 (s, 1H, NH; exchangeable with $\mathrm{D}_{2} \mathrm{O}$ ); Anal. Calcd for $\mathrm{C}_{18} \mathrm{H}_{22} \mathrm{~N}_{2} \mathrm{O}_{6}$ (362.38): C, 59.66; H, 6.12; N, 7.73 Found: C, 59.50; H, 5.96; N, 7.60.

5-(1,2,3,4-Tetrahydroxybutyl)-N-(1-(4-hydroxyphenyl)ethylidene)-2-methylfuran-3-carbohydrazide (4). Yield 63.8\%. Recrystallized from ethanol as yellow crystals; m.p. $229-230{ }^{\circ} \mathrm{C} ; \quad \mathrm{R}_{\mathrm{f}}: 0.76$ $\left(\mathrm{CHCl}_{3} / \mathrm{MeOH}, 20: 1, \mathrm{v} / \mathrm{v}\right)[\alpha]_{\mathrm{D}}{ }^{20}-5.5$; IR $(\mathrm{KBr}): 1599(\mathrm{C}=\mathrm{N}), 1655(\mathrm{CONH}), 3254,3322 \mathrm{~cm}^{-1}(\mathrm{NH}$ and $\mathrm{OH}$ ); ${ }^{1} \mathrm{H}-\mathrm{NMR}$ (DMSO- $d_{6}$ ) $\delta: 2.08$ (s, 3H, $\mathrm{CH}_{3} \mathrm{CN}$ ), 2.21 (s, 3H, $\mathrm{CH}_{3}$-furan), 2.51-2.54 (m, 4H, H-2', H-3', H-4a', H-4b'), 3.42 (bs, 1H, 5'-OH; exchangeable with $\mathrm{D}_{2} \mathrm{O}$ ), 4.33-4.34 (m, 1H, 4'-OH; exchangeable with $\left.\mathrm{D}_{2} \mathrm{O}\right), 4.43\left(\mathrm{dd}, 1 \mathrm{H}, 3^{\prime}-\mathrm{OH} ; J_{1,2}=7.7 \mathrm{~Hz}, J_{1,3}=16.8 \mathrm{~Hz}\right.$; exchangeable with $\left.\mathrm{D}_{2} \mathrm{O}\right)$, 4.57-4.59 (m, 1H, 2'-OH; exchangeable with $\left.\mathrm{D}_{2} \mathrm{O}\right), 4.70$ (dd, $1 \mathrm{H}, \mathrm{H}-1$ '; $J_{1,2}=6.1 \mathrm{~Hz}, J_{1,3}=15.3 \mathrm{~Hz}$ ), $5.06\left(\mathrm{~d}, 1 \mathrm{H}, 1^{\prime}-\mathrm{OH} ; J=6.9 \mathrm{~Hz}\right.$, exchangeable with $\left.\mathrm{D}_{2} \mathrm{O}\right), 6.69$ (s, 1H, H-furan), 6.79 (d, 2H, o-OH; $J=8.4 \mathrm{~Hz}), 7.73(\mathrm{~d}, 2 \mathrm{H}, m-\mathrm{OH} ; J=8.4 \mathrm{~Hz}), 9.73\left(\mathrm{bs}, 1 \mathrm{H}, \mathrm{NH}\right.$; exchangeable with $\left.\mathrm{D}_{2} \mathrm{O}\right)$; Anal. Calcd for $\mathrm{C}_{18} \mathrm{H}_{22} \mathrm{~N}_{2} \mathrm{O}_{7}$ (378.38): C, 57.14; H, 5.86; N, 7.40 Found: C, 57.29; H, 6.00; N, 7.56.

N'-(1-(4-Aminophenyl)ethylidene))-5-(1,2,3,4-tetrahydroxybutyl)-2-methylfuran-3-carbohydrazide (5). Yield 84.9\%. Recrystallized from ethanol as golden crystals; m.p. $174-175{ }^{\circ} \mathrm{C} ; \quad \mathrm{R}_{\mathrm{f}}: 0.83$ $\left(\mathrm{CHCl}_{3} / \mathrm{MeOH}, 20: 1, \mathrm{v} / \mathrm{v}\right)$; $[\alpha]_{\mathrm{D}}{ }^{20}-17$; IR $(\mathrm{KBr}): 1588(\mathrm{C}=\mathrm{N}), 1644(\mathrm{CONH}), 3238,3334,3387 \mathrm{~cm}^{-1}$ $\left(\mathrm{NH}, \mathrm{OH}\right.$, and $\left.\mathrm{NH}_{2}\right)$; ${ }^{1} \mathrm{H}-\mathrm{NMR}\left(\mathrm{DMSO}-d_{6}\right) \delta: 2.19$ (s, 3H, $\left.\mathrm{CH}_{3} \mathrm{CN}\right), 2.44$ (s, 3H, $\mathrm{CH}_{3}$-furan), 3.33-3.38 (m, 1H, H-3'), 3.41-3.44 (m, 1H, H-2'), 3.47-3.49 (m, 1H, H-4a'), 3.50-3.53 (m, 1H, H-4b'), 4.28-4.33 $\left(\mathrm{m}, 1 \mathrm{H}, 4\right.$ '-OH; exchangeable with $\left.\mathrm{D}_{2} \mathrm{O}\right), 4.41\left(\mathrm{~d}, 1 \mathrm{H}, 3\right.$ '- $\mathrm{OH} ; J=7.7 \mathrm{~Hz}$, exchangeable with $\left.\mathrm{D}_{2} \mathrm{O}\right)$, $4.56\left(\mathrm{~d}, 1 \mathrm{H}, 2^{\prime}-\mathrm{OH} ; J=3.4 \mathrm{~Hz}\right.$, exchangeable with $\left.\mathrm{D}_{2} \mathrm{O}\right), 4.68$ (d, 1H, H-1'; $\left.J=4.6 \mathrm{~Hz}\right), 5.05$ (d, 1H, 1'-OH; $J=6.8 \mathrm{~Hz}$, exchangeable with $\left.\mathrm{D}_{2} \mathrm{O}\right), 5.44\left(\mathrm{~s}, 2 \mathrm{H}, \mathrm{NH}_{2}\right.$; exchangeable with $\left.\mathrm{D}_{2} \mathrm{O}\right), 6.54(\mathrm{~d}, 2 \mathrm{H}$, $\left.o-\mathrm{NH}_{2}\right), 6.57$ (s, 1H, H-furan), $7.58\left(\mathrm{~d}, 2 \mathrm{H}, m-\mathrm{NH}_{2}\right), 9.20\left(\mathrm{bs}, 1 \mathrm{H}, \mathrm{NH}\right.$; exchangeable with $\left.\mathrm{D}_{2} \mathrm{O}\right)$; MS: m/z (\%), 77 (4.54), 92 (53.25), 118 (67.81), 133 (41.59), 149 (8.57), 210 (9.36), 251 (100), 252 (17.94), 266 (68.55), 267 (13.58, $\left.\mathrm{M}^{+}\right)$; Anal. Calcd for $\mathrm{C}_{18} \mathrm{H}_{23} \mathrm{~N}_{3} \mathrm{O}_{6}$ (377.39): C, 57.29; H, 6.14; N, 11.13 Found: C, 57.40; H, 6.29; N, 11.21. 
5-(1,2,3,4-Tetrahydroxybutyl)-2-methyl-N-(4-methylpentane-2-ylidene)furan-3-carbohydrazide

Yield $82 \%$. Recrystallized from ethanol as white crystals; m.p. $142-143{ }^{\circ} \mathrm{C} ; \mathrm{R}_{\mathrm{f}}: 0.55\left(\mathrm{CHCl}_{3} / \mathrm{MeOH}\right.$, 20:1, v/v); $[\alpha]_{\mathrm{D}}{ }^{20}-8.9$; IR ( $\left.\mathrm{KBr}\right): 1581(\mathrm{C}=\mathrm{N}), 1651(\mathrm{CONH}), 3260(\mathrm{NH}), 3321 \mathrm{~cm}^{-1}(\mathrm{OH}) ;{ }^{1} \mathrm{H}-\mathrm{NMR}$ (DMSO-d $\left.)_{6}\right) \delta: 0.85\left(\mathrm{~d}, 6 \mathrm{H}, 2 \mathrm{CH}_{3} ; J=6.9 \mathrm{~Hz}\right), 1.83\left(\mathrm{~s}, 3 \mathrm{H}, \mathrm{CH}_{3} \mathrm{CN}\right), 1.89-1.90\left(\mathrm{~m}, 1 \mathrm{H}, \mathrm{CH}\left(\mathrm{CH}_{3}\right)_{2}\right)$, 2.08 (m, 2H, $\mathrm{CH}_{2}$ ), 2.44 (s, 3H, $\mathrm{CH}_{3}$-furan), 3.44-3.54 (m, 4H, H-2', H-3', H-4a', H-4b'), 4.34 (d, 1H, 4'-OH; $J=5.4 \mathrm{~Hz}$, exchangeable with $\left.\mathrm{D}_{2} \mathrm{O}\right), 4.43\left(\mathrm{dd}, 1 \mathrm{H}, 3^{\prime}-\mathrm{OH} ; J_{1,2}=7.7 \mathrm{~Hz}, J_{1,3}=16.8 \mathrm{~Hz}\right.$; exchangeable with $\left.\mathrm{D}_{2} \mathrm{O}\right), 4.58\left(\mathrm{t}, 1 \mathrm{H}, 2^{\prime}-\mathrm{OH} ; J_{1,2}=7.7 \mathrm{~Hz}, J_{1,3}=13.8 \mathrm{~Hz}\right.$; exchangeable with $\left.\mathrm{D}_{2} \mathrm{O}\right)$, $4.70\left(\mathrm{dd}, 1 \mathrm{H}, \mathrm{H}-1\right.$ '; $\left.J_{1,2}=6.1 \mathrm{~Hz}, J_{1,3}=15.3 \mathrm{~Hz}\right), 5.06$ (d, 1H, 1'-OH; $J=6.9 \mathrm{~Hz}$, exchangeable with $\left.\mathrm{D}_{2} \mathrm{O}\right), 6.65(\mathrm{~d}, 1 \mathrm{H}, \mathrm{H}$-furan; $J=12.3 \mathrm{~Hz}), 9.87\left(\mathrm{~s}, 1 \mathrm{H}, \mathrm{NH}\right.$; exchangeable with $\left.\mathrm{D}_{2} \mathrm{O}\right)$; $\mathrm{MS}: \mathrm{m} / z(\%), 55$ (15.90), 57 (46.21), 69 (7.03), 71 (25.45), 95 (6.39), 96 (6.09), 111 (5.61), 113 (14.11), 139 (8.44), 149 (100), 150 (11.54), 167 (30.60), 168 (2.57), 185 (2.07), 230 (2.43), 284 (1.22), 342 (3.66, M ). Anal. Calcd for $\mathrm{C}_{16} \mathrm{H}_{26} \mathrm{~N}_{2} \mathrm{O}_{6}$ (342.39): C, 56.13; H, 7.65; N, 8.18 Found: C, 56.29; H, 7.44; N, 8.30.

\subsection{Reactions of 3-6 with Acetic Anhydride}

5-(1,2,3,4-Tetrahydroxybutyl)-2-methyl-N-(1-arylethylidene)furan-3-carbohydrazides 3-5 (0.002 mol) were dissolved in pyridine $(10 \mathrm{~mL})$ and acetic anhydride $(10 \mathrm{~mL})$ and left for $24 \mathrm{~h}$. The mixture was then poured onto crushed ice, the product that separated was filtered off, washed several times with water and dried.

5-(1,2,3,4-Tetracetoxybutyl)-2-methyl-N-(1-phenylethylidene)furan-3-carbohydrazide (7). Yield 86\%. Recrystallized from ethanol as yellow crystals; m.p. $134-135{ }^{\circ} \mathrm{C}, \mathrm{R}_{\mathrm{f}}: 0.83\left(\mathrm{CHCl}_{3} / \mathrm{MeOH}, 20: 1\right.$, v/v); $[\alpha]_{\mathrm{D}}{ }^{20}-12.9$; IR $(\mathrm{KBr}): 1594(\mathrm{C}=\mathrm{N}), 1654(\mathrm{CONH}), 1720\left(\mathrm{CO}\right.$-acetyl), $3362 \mathrm{~cm}^{-1}(\mathrm{NH})$; Anal. Calcd for $\mathrm{C}_{26} \mathrm{H}_{30} \mathrm{~N}_{2} \mathrm{O}_{10}(530.52)$ : C, 58.86; H, 5.70; N, 5.28 Found: C, 58.72; H, 5.52; N, 4.99.

5-(1,2,3,4-Tetraacetoxybutyl)-N-(1-(4-acetoxyphenyl)ethylidene)-2-methylfuran-3-carbohydrazide (8). Yield 44.9\%. Recrystallized from ethanol as pale yellow crystals; m.p. $149-150{ }^{\circ} \mathrm{C} ; \mathrm{R}_{\mathrm{f}}: 0.24$ $\left(\mathrm{CHCl}_{3} / \mathrm{MeOH}, 25: 1, \mathrm{v} / \mathrm{v}\right)$; $[\alpha]_{\mathrm{D}}{ }^{20}-14.2$; IR $(\mathrm{KBr}): 1579(\mathrm{C}=\mathrm{N}), 1635$ (CONH), 1754 (CO-acetyl), $3311 \mathrm{~cm}^{-1}(\mathrm{NH}) ;{ }^{1} \mathrm{H}-\mathrm{NMR}\left(\mathrm{CHCl}_{3}-d\right) \delta: 2.17\left(\mathrm{~s}, 3 \mathrm{H}, \mathrm{CH}_{3} \mathrm{CO}\right), 2.31(\mathrm{~d}, 12 \mathrm{H}, 4 \mathrm{O}-\mathrm{Ac}), 2.43(\mathrm{~s}, 3 \mathrm{H}$,

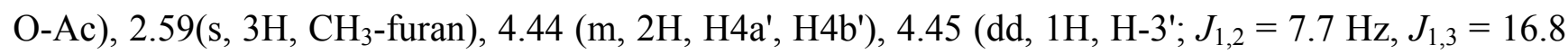
$\mathrm{Hz}), 4.47$ (t, 1H, H-2'; $\left.J_{1,2}=7.7 \mathrm{~Hz}, J_{1,3}=13.8 \mathrm{~Hz}\right), 4.58\left(\mathrm{dd}, 1 \mathrm{H}, \mathrm{H}-1^{\prime} ; J_{1,2}=6.1 \mathrm{~Hz}, J_{1,3}=15.3 \mathrm{~Hz}\right)$, 6.64 (s, 1H, H-furan), 7.14 (d, 2H,o-OAc), 7.93 (d, 2H, m-OAc), (s, 1H, NH; exchangeable with $\mathrm{D}_{2} \mathrm{O}$ ); Anal. Calcd for $\mathrm{C}_{28} \mathrm{H}_{32} \mathrm{~N}_{2} \mathrm{O}_{12}(588.56)$ : C, 57.14; H, 5.48; N, 4.76 Found: C, 57.28; H, 5.60; N, 4.89 .

$N^{\prime}$-(1-(4-Aminophenyl)ethylidene))-5-(1,2,3,4-tetracetoxybutyl)-2-methylfuran-3-carbohydrazide

Yield $60.5 \%$, Recrystallized from ethanol as yellow crystals; m.p. $279-280{ }^{\circ} \mathrm{C}, \mathrm{R}_{\mathrm{f}}$ : $\left(\mathrm{CHCl}_{3} / \mathrm{MeOH}\right.$, 20:1, v/v); $[\alpha]_{\mathrm{D}}{ }^{20}-6.8$; IR $(\mathrm{KBr}): 1602(\mathrm{C}=\mathrm{N}), 1660(\mathrm{CONH}), 1732\left(\mathrm{CO}\right.$-acetyl), $3288 \mathrm{~cm}^{-1}(\mathrm{NH})$;

${ }^{1} \mathrm{H}-\mathrm{NMR}\left(\mathrm{DMSO}-d_{6}\right) \delta: 2.03$ (s, 6H, 2CH ), 2.23 (s, 18H, 4O-Ac, N-Ac), 3.34-3.54 (m, 4H, H-1',H-2', H-3', H4a,4b'), 6.64 (s, 1H, H-furan), 7.62 (d, 2H, Ar-H; J=8.4 Hz), 7.82 (d, 2H, Ar-H; J= 8.4 Hz), $9.03\left(\mathrm{~s}, 1 \mathrm{H}, \mathrm{NH}\right.$; exchangeable with $\left.\mathrm{D}_{2} \mathrm{O}\right), 10.08$ (s, $1 \mathrm{H}, \mathrm{NH}$; exchangeable with $\left.\mathrm{D}_{2} \mathrm{O}\right)$. Anal. Calcd for $\mathrm{C}_{28} \mathrm{H}_{33} \mathrm{~N}_{3} \mathrm{O}_{11}$ (587.58): C, 57.24; H, 5.66; N, 7.15 Found: C, 57.02; H, 5.49; N, 7.02. 


\subsection{Periodate Oxidation of $\mathbf{3}-\mathbf{6}$}

A solution of 3-6 $(0.003 \mathrm{~mol})$ dissolved in distilled water $(50 \mathrm{~mL})$ was treated with a solution of $\mathrm{NaIO}_{4}(0.008 \mathrm{~mol})$ in distilled water $(50 \mathrm{~mL})$ dropwise with stirring for $3 \mathrm{~h}$, the product that separated out was filtered off, washed with water and dried.

5-Formyl-2-methyl-N'-(1-phenylethylidene) furan-3-carbohydrazide (10). Yield 65\%. Recrystallized from $\mathrm{EtOH}$ as a yellow powder; m.p. $114-115^{\circ} \mathrm{C}$; $\mathrm{R}_{\mathrm{f}}: 0.84\left(\mathrm{CHCl}_{3} / \mathrm{MeOH}, 20: 1, \mathrm{v} / \mathrm{v}\right)$; IR (KBr): 1593 $(\mathrm{C}=\mathrm{N}), 1643(\mathrm{CONH}), 1728(\mathrm{CHO}), 3236 \mathrm{~cm}^{-1}(\mathrm{NH}) ;{ }^{1} \mathrm{H}-\mathrm{NMR}\left(\mathrm{DMSO}-d_{6}\right) \delta: 2.23\left(\mathrm{~s}, 6 \mathrm{H}, 2 \mathrm{CH}_{3}\right)$, 7.42-7.43 (m, 2H, Ar-H), 7.31 (s, 1H, H-furan), 7.87-7.93 (m, 3H, Ar-H), 9.23 (bs, 1H, NH; exchangeable with $\mathrm{D}_{2} \mathrm{O}$ ), 9.87 (s, $1 \mathrm{H}, \mathrm{CHO}$ ); Anal. Calcd for $\mathrm{C}_{15} \mathrm{H}_{14} \mathrm{~N}_{2} \mathrm{O}_{3}$ (270.28): C, 66.66; H, 5.22; N, 10.36 Found: C, 66.44; H, 4.99; N, 10.19.

N'-(1-(4-Hydroxyphenyl)ethylidene)-5-formyl-2-methylfuran-3-carbohydrazide (11). Yield 43.5\%. Recrystallized from EtOH as white crystals; m.p. $192-193{ }^{\circ} \mathrm{C} ; \mathrm{R}_{\mathrm{f}}: 0.32\left(\mathrm{CHCl}_{3} / \mathrm{MeOH}, 25: 1\right.$, v/v); IR $(\mathrm{KBr}): 1597(\mathrm{C}=\mathrm{N}), 1668(\mathrm{CONH}), 1751(\mathrm{CHO}), 3251,3404 \mathrm{~cm}^{-1}(\mathrm{NH}$ and $\mathrm{OH}) ;{ }^{1} \mathrm{H}-\mathrm{NMR}\left(\mathrm{DMSO}-d_{6}\right)$ $\delta: 2.27\left(\mathrm{~s}, 6 \mathrm{H}, 2 \mathrm{CH}_{3}\right), 4.91\left(\mathrm{bs}, 1 \mathrm{H}, \mathrm{OH}\right.$; exchangeable with $\left.\mathrm{D}_{2} \mathrm{O}\right), 6.86(\mathrm{~d}, 2 \mathrm{H}, o-\mathrm{OH} ; J=8.4 \mathrm{~Hz})$, 7.81(d, 2H, $m-\mathrm{OH} ; J=8.4 \mathrm{~Hz}), 8.91$ (s, $1 \mathrm{H}, \mathrm{NH}$; exchangeable with $\left.\mathrm{D}_{2} \mathrm{O}\right), 9.34$ (s, 1H, CHO); Anal. Calcd for $\mathrm{C}_{15} \mathrm{H}_{14} \mathrm{~N}_{2} \mathrm{O}_{4}$ (286.28): C, 62.93; H, 4.93; N, 9.79 Found: C, 62.75; H, 4.77; N, 9.60.

$N^{\prime}$-(1-(4-Aminophenyl)ethylidene)-5-formyl-2-methylfuran-3-carbohydrazide (12). Yield $\quad 36 \%$. Rerystallized from EtOH as dark yellow crystals; m.p. $145-146{ }^{\circ} \mathrm{C} ; \mathrm{R}_{\mathrm{f}}: 0.86\left(\mathrm{CHCl}_{3} / \mathrm{MeOH}, 20: 1\right.$, $\mathrm{v} / \mathrm{v})$; IR (KBr): $1586(\mathrm{C}=\mathrm{N}), 1652(\mathrm{CONH}), 1768(\mathrm{CHO}), 3233,3322,3344 \mathrm{~cm}^{-1}\left(\mathrm{NH}\right.$ and $\left.\mathrm{NH}_{2}\right)$; ${ }^{1} \mathrm{H}-\mathrm{NMR}\left(\mathrm{DMSO}-d_{6}\right) \delta: 2.27\left(\mathrm{~s}, 6 \mathrm{H}, 2 \mathrm{CH}_{3}\right), 4.95\left(\mathrm{bs}, 2 \mathrm{H}, \mathrm{NH}_{2}\right)$; exchangeable with $\left.\mathrm{D}_{2} \mathrm{O}\right), 6.65-6.70$ $\left(\mathrm{m}, 2 \mathrm{H}, o-\mathrm{NH}_{2}\right), 6.58\left(\mathrm{~s}, 1 \mathrm{H}, \mathrm{H}\right.$-furan), 7.69-7.73 (m, 2H, $\left.m-\mathrm{NH}_{2}\right), 9.36(\mathrm{~s}, 1 \mathrm{H}, \mathrm{NH}$; exchangeable with $\mathrm{D}_{2} \mathrm{O}$ ), 9.44 (s, 1H, CHO); Anal. Calcd for $\mathrm{C}_{15} \mathrm{H}_{15} \mathrm{~N}_{3} \mathrm{O}_{3}$ (285.3): C, 63.15; H, 5.30; N, 14.73 Found: C, $62.99 ; \mathrm{H}, 5.22 ; \mathrm{N}, 14.62$.

5-Formyl-2-methyl-N'-(4-methylpentan-2-ylidene)furan-3-carbohydrazide $\quad(\mathbf{1 3}) . \quad$ Yield $\quad 58 \%$. Recrystallized from EtOH as pale yellow needles; m.p. $210-211{ }^{\circ} \mathrm{C}$; $\mathrm{R}_{\mathrm{f}}: 0.77$ (n-hexane/EtOAc, 7:1, v/v); IR (KBr): $1632(\mathrm{C}=\mathrm{N}), 1666(\mathrm{CONH}), 1720(\mathrm{CHO}), 3437 \mathrm{~cm}^{-1}(\mathrm{NH}) ; \mathrm{MS}: \mathrm{m} / z(\%), 65(48.47)$, 80 (33.29), 92 (40.53), 93 (6.69), 104 (7.80), 113 (5.29), 117 (11), 118 (72.01), 119 (33.98), 122 (8.50), 132 (11.42), 133 (51.67), 136 (4.46), 141 (16.43), 145 (14.62), 148 (32.17), 149 (80.22), 150 (5.99), 158 (16.85), 167 (23.68), 174 (12.26), 178 (17.27), 181 (39.42), 182 (23.54), 193 (16.16), 195 (17.97), 196 (15.46), 211 (17.97), 224 (18.11), 225 (15.32), 227 (15.46), 230 (15.88), $250\left(100, \mathrm{M}^{+}\right)$; Anal. Calcd for $\mathrm{C}_{13} \mathrm{H}_{18} \mathrm{~N}_{2} \mathrm{O}_{3}$ (250.29): C, 62.38; H, 7.25; N, 11.19 Found: C, 62.13; H, 7.02; N, 11.10.

\subsection{Reactions of 5-Formyl-2-methyl-N'-(1-arylethylidene) furan-3-carbohydrazide 10-12 with} Thio-semicarbazide Derivatives

A solution of 5-formyl-2-methyl-N'-(1-arylethylidene)furan-3-carbohydrazide 10-12 (0.001 mol) in ethanol $(20 \mathrm{~mL})$ containing acetic acid $(0.01 \mathrm{~mL})$ was treated with thiosemicarbazide or $p$-tolyl- or $o$-tolylthiosemicarbazide $(0.001 \mathrm{~mol})$. The mixture was refluxed for 3-6 h. After cooling, the thiosemicarbazone which separated out was filtered off, washed with little ethanol and dried. 
1-((4-(1-Phenylethylideneaminocarbamoyl)-5-methylfuran-2-yl)methylene)thiosemicarbazide

Yield 98\%. Recrystallized from ethanol as yellow needles; m.p. $159-160{ }^{\circ} \mathrm{C} ; \mathrm{R}_{\mathrm{f}}: 0.83\left(\mathrm{CHCl}_{3} / \mathrm{MeOH}\right.$, 20:1, v/v); IR (KBr): 1489 (CSNH), $1589(\mathrm{C}=\mathrm{N}), 1684(\mathrm{CONH}), 3148,3207(2 \mathrm{NH}), 3362,3405 \mathrm{~cm}^{-1}$ $\left(\mathrm{NH}_{2}\right) ;{ }^{1} \mathrm{H}-\mathrm{NMR}\left(\mathrm{CHCl}_{3}-d\right) \delta: 1.83\left(\mathrm{bs}, 2 \mathrm{H}, \mathrm{NH}_{2}\right.$; exchangeable with $\left.\mathrm{D}_{2} \mathrm{O}\right), 2.29\left(\mathrm{~s}, 3 \mathrm{H}, \mathrm{CH}_{3} \mathrm{CN}\right), 2.31$ (s, 3H, $\mathrm{CH}_{3}$-furan), 6.51 (bs, $1 \mathrm{H}, \mathrm{NH}$; exchangeable with $\left.\mathrm{D}_{2} \mathrm{O}\right), 7.35-7.47$ (m, 5H, Ar-H), 7.69 (s, 1H, $\mathrm{H}$-furan), $7.91(\mathrm{~s}, 1 \mathrm{H}, \mathrm{CH}=\mathrm{N}), 8.79\left(\mathrm{~s}, 1 \mathrm{H}, \mathrm{NH}\right.$; exchangeable with $\left.\mathrm{D}_{2} \mathrm{O}\right) ; \mathrm{MS}: m / z(\%), 51$ (21.35), 76 (6.26), 77 (82.55), 91 (9.79), 92 (4.78), 103 (22.11), 118 (32.79), 133 (11.33), 221 (100), 222 (21.64), 343 (19.01, $\mathrm{M}^{+}$); Anal. Calcd for $\mathrm{C}_{16} \mathrm{H}_{17} \mathrm{~N}_{5} \mathrm{O}_{2} \mathrm{~S}$ (343.4): C, 55.96; H, 4.99; N, 20.39 Found: C, 55.79; $\mathrm{H}, 4.84 ; \mathrm{N}, 20.22$.

1-((4-(1-(4-Hydroxyphenylethylideneaminocarbamoyl)furan-2-yl)methylene)thiosemi-carbazide (15). Yield 99.0\%. Recrystallized from ethanol as yellow crystals; m.p. $146-147{ }^{\circ} \mathrm{C} ; \mathrm{R}_{\mathrm{f}}$ : 0.47 $\left(\mathrm{CHCl}_{3} / \mathrm{MeOH}, 25: 1, \mathrm{v} / \mathrm{v}\right)$; IR (KBr): $1499(\mathrm{CSNH}), 1585(\mathrm{C}=\mathrm{N}), 1654(\mathrm{CONH}), 3182,3200,3358$ $\mathrm{cm}^{-1}\left(2 \mathrm{NH}, \mathrm{NH}_{2}\right.$, and $\left.\mathrm{OH}\right) ;{ }^{1} \mathrm{H}-\mathrm{NMR}\left(\mathrm{DMSO}-d_{6}\right) \delta: 2.19\left(\mathrm{~s}, 6 \mathrm{H}, 2 \mathrm{CH}_{3}\right), 4.34\left(\mathrm{bs}, 2 \mathrm{H}, \mathrm{NH}_{2}\right.$; exchangeable with $\left.\mathrm{D}_{2} \mathrm{O}\right), 6.72(\mathrm{~d}, 2 \mathrm{H}, o-\mathrm{OH} ; J=8.4 \mathrm{~Hz}), 6.76(\mathrm{~d}, 1 \mathrm{H}, \mathrm{H}-$ furan; $J=6.9 \mathrm{~Hz}), 7.73(\mathrm{~d}$, $2 \mathrm{H}, m-\mathrm{OH} ; J=8.4 \mathrm{~Hz}), 7.77(\mathrm{~s}, 1 \mathrm{H}, \mathrm{CH}=\mathrm{N}), 8.12\left(\mathrm{~s}, 1 \mathrm{H}, \mathrm{OH}\right.$; exchangeable with $\left.\mathrm{D}_{2} \mathrm{O}\right), 9.73(\mathrm{bs}, 1 \mathrm{H}$, $\mathrm{NH}$; exchangeable with $\left.\mathrm{D}_{2} \mathrm{O}\right), 10.02\left(\mathrm{~s}, 1 \mathrm{H}, \mathrm{NH}\right.$; exchangeable with $\left.\mathrm{D}_{2} \mathrm{O}\right)$; Anal. Calcd for $\mathrm{C}_{16} \mathrm{H}_{17} \mathrm{~N}_{5} \mathrm{O}_{3} \mathrm{~S}$ (359.4): C, 53.47; H, 4.77; N, 19.49 Found: C, 53.36; H, 4.59; N, 19.34.

1-((4-(1-(4-Aminophenylethylideneaminocarbamoyl)furan-2-yl)methylene)thiosemicarbazide

(16).

Yield $43 \%$. Recrystallized from ethanol as orange crystals; m.p. $179-180{ }^{\circ} \mathrm{C} ; \mathrm{R}_{\mathrm{f}}: 0.75\left(\mathrm{CHCl}_{3} / \mathrm{MeOH}\right.$, 20:1, v/v); IR (KBr): $1489(\mathrm{CSNH}), 1591(\mathrm{C}=\mathrm{N}), 1652(\mathrm{CONH}), 3311,3388,3344 \mathrm{~cm}^{-1}\left(\mathrm{NH}, \mathrm{NH}_{2}\right)$; ${ }^{1} \mathrm{H}-\mathrm{NMR}\left[\left(\mathrm{CH}_{3}\right)_{2} \mathrm{CO}-d_{6}\right] \delta: 2.28\left(\mathrm{~s}, 6 \mathrm{H}, 2 \mathrm{CH}_{3}\right), 4.99\left(\mathrm{bs}, 4 \mathrm{H}, 2 \mathrm{NH}_{2}\right.$; exchangeable with $\left.\mathrm{D}_{2} \mathrm{O}\right), 6.61(\mathrm{~d}$, $\left.2 \mathrm{H}, o-\mathrm{NH}_{2} ; J=8.4 \mathrm{~Hz}\right), 6.63\left(\mathrm{~s}, 1 \mathrm{H}, \mathrm{H}\right.$-furan), $7.40\left(\mathrm{bs}, 1 \mathrm{H}, \mathrm{NH}\right.$; exchangeable with $\left.\mathrm{D}_{2} \mathrm{O}\right), 7.61(\mathrm{~d}, 2 \mathrm{H}$, $\left.m-\mathrm{NH}_{2} ; J=8.4 \mathrm{~Hz}\right), 7.70(\mathrm{~s}, 1 \mathrm{H}, \mathrm{CH}=\mathrm{N}), 9.23\left(\mathrm{~s}, 1 \mathrm{H}, \mathrm{NH}\right.$; exchangeable with $\left.\mathrm{D}_{2} \mathrm{O}\right) ; \mathrm{MS}: m / z(\%), 64$ (7.85), 65 (62.80), 77 (5.46), 80 (5.78), 91(26.67), 92 (55.68), 106 (7.60), 107 (7.39), 118 (71.91), 119 (38.57), 133 (61.38), 134 (17.57), 148 (15.14), 174 (4.71), 191 (27.20), 208 (30.73), 209 (4.70), 210 (9.01), 251 (100), 252 (18.49), 266 (66.40),358 (17.57, $\mathrm{M}^{+}$); Anal. Calcd for $\mathrm{C}_{16} \mathrm{H}_{18} \mathrm{~N}_{6} \mathrm{O}_{2} \mathrm{~S}$ (358.42): C, 53.62; H, 5.06; N, 23.45 Found: C, 53.41; H, 4.97; N, 23.22.

1-((4-(1-Phenylethylideneaminocarbamoyl)-5-methylfuran-2-yl)methylene)-4-o-tolylthiosemicarbazide (17). Yield 68.8\%/ Recrystallized from ethanol as white needles; m.p. 157-158 ${ }^{\circ} \mathrm{C}$; $\mathrm{R}_{\mathrm{f}}$ : 0.91 $\left(\mathrm{CHCl}_{3} / \mathrm{MeOH}, 20: 1, \mathrm{v} / \mathrm{v}\right)$; IR $(\mathrm{KBr}): 1488(\mathrm{CSNH}), 1602(\mathrm{C}=\mathrm{N}), 1658(\mathrm{CONH}), 3220,3293 \mathrm{~cm}^{-1}$ $(2 \mathrm{NH}) ;{ }^{1} \mathrm{H}-\mathrm{NMR}\left(\mathrm{CHCl}_{3}-d\right) \delta: 2.35\left(\mathrm{~s}, 9 \mathrm{H}, 3 \mathrm{CH}_{3}\right), 7.21(\mathrm{~s}, 1 \mathrm{H}, \mathrm{H}-$ furan$), 7.23(\mathrm{~s}, 1 \mathrm{H}, \mathrm{CH}=\mathrm{N})$, 7.25-7.27 (m, 2H, Ar-H), 7.41-7.46 (m, 4H, Ar-H), 7.73-7.75 (m, 3H, Ar-H), 8.96 (s, 1H, NH; exchangeable with $\left.\mathrm{D}_{2} \mathrm{O}\right), 9.21\left(\mathrm{~s}, 2 \mathrm{H}, 2 \mathrm{NH}\right.$; exchangeable with $\left.\mathrm{D}_{2} \mathrm{O}\right)$; $\mathrm{MS}: m / z(\%), 65(28.65), 77$ (100), 91 (32.72), 103 (16.00), 106 (25.49), 107 (51.97), 118 (24.99), 133 (64.04), 134 (19.96), 150 (15.79), 151 (9.96), 164 (11.37), 165 (8.88), 268 (61.82), 283 (52.96), 284 (10.21), 433 (8.86, M ); Anal. Calcd for $\mathrm{C}_{23} \mathrm{H}_{23} \mathrm{~N}_{5} \mathrm{O}_{2} \mathrm{~S}$ (433.53): C, 63.72; H, 5.35; N, 16.15 Found: C, 63.47; H, 5.11; N, 15.90 .

1-((4-(1-(4-Hydroxyphenylethylideneaminocarbamoyl)-5-methylfuran-2-yl)methylene)-4-o-tolylthiosemicarbazide (18). Yield 53\%. Recrystallized from ethanol as yellow crystals; m.p. $229-230{ }^{\circ} \mathrm{C}$; $\mathrm{R}_{\mathrm{f}}$ : 
$0.5\left(\mathrm{CHCl}_{3} / \mathrm{MeOH}, 25: 1, \mathrm{~V} / \mathrm{V}\right)$; IR (KBr): $1486(\mathrm{CSNH}), 1613(\mathrm{C}=\mathrm{N}), 1664(\mathrm{CONH}), 3235,3323$, $3462 \mathrm{~cm}^{-1}(2 \mathrm{NH}, \mathrm{OH}){ }^{1} \mathrm{H}-\mathrm{NMR}$ (DMSO-d $) \delta: 2.19$ (s, 3H, $\mathrm{CH}_{3} \mathrm{CN}$ ), 2.21 (s, 3H, $\mathrm{CH}_{3}$-fursn), 2.28 (s, $3 \mathrm{H}, \mathrm{CH}_{3}$-tolyl), 6.74 (s,1H, H-furan), $5.68\left(\mathrm{~s}, 1 \mathrm{H}, \mathrm{OH}\right.$; exchangeable with $\left.\mathrm{D}_{2} \mathrm{O}\right), 6.79$ (d, 2H,o-OH; $J=8.4 \mathrm{~Hz}$ ), 7.13-7.20 (m, 2H, o-tolyl), 7.23 (d, 1H, o-tolyl; $J=6.9 \mathrm{~Hz}), 7.32$ (d, 1H, o-tolyl; $J=7.7 \mathrm{~Hz}), 7.73(\mathrm{~d}, 2 \mathrm{H}, m-\mathrm{OH} ; J=8.4 \mathrm{~Hz}), 7.84(\mathrm{~s}, 1 \mathrm{H}, \mathrm{CH}=\mathrm{N}), 9.78$ (bs, 3H, 3NH; exchangeable with $\left.\mathrm{D}_{2} \mathrm{O}\right)$; MS: $m / z(\%), 50$ (10.85), 51 (24.80), 65 (90.46), 77 (74.51), 91 (70.32), 107 (100), 119 (51.20), 134 (57.54), 149 (28.11), 150 (15.98), 164 (10.61), 175 (10.30), 205 (6.06), 212 (8.73), 237 (6.29), 253 (97.99), 268 (80.70), 283 (26.17), 284 (9.88), 296 (6.67), 299 (6.04), 449 (10.61, M ); Anal. Calcd for $\mathrm{C}_{23} \mathrm{H}_{23} \mathrm{~N}_{5} \mathrm{O}_{3} \mathrm{~S}$ (449.53): C, 61.45; H, 5.16; N, 15.58 Found: C, 61.23; H, 5.02; N, 15.40.

1-((4-(1-(4-Aminophenylethylideneaminocarbamoyl)-5-methylfuran-2-yl)methylene)-4-o-tolylthiosemicarbazide (19). Yield 44\%. Recrystallized from ethanol as yellow crystals; m.p. $139-140{ }^{\circ} \mathrm{C}$; $\mathrm{R}_{\mathrm{f}}$ : $0.77\left(\mathrm{CHCl}_{3} / \mathrm{MeOH}, 20: 1, \mathrm{v} / \mathrm{v}\right)$; IR (KBr): $1482(\mathrm{CSNH}), 1622(\mathrm{C}=\mathrm{N}), 1683(\mathrm{CONH}), 3205,3252$, $3288,3324 \mathrm{~cm}^{-1}\left(3 \mathrm{NH}, \mathrm{NH}_{2}\right)$; ${ }^{1} \mathrm{H}-\mathrm{NMR}\left[\left(\mathrm{CH}_{3}\right)_{2} \mathrm{CO}-d_{6}\right] \delta: 2.20\left(\mathrm{~s}, 3 \mathrm{H}, \mathrm{CH}_{3} \mathrm{CN}\right), 2.24(\mathrm{~s}, 3 \mathrm{H}$, $\mathrm{CH}_{3}$-furan), 2.35 (s, $3 \mathrm{H}, \mathrm{CH}_{3}$-tolyl), 5.45 (bs, $2 \mathrm{H}, \mathrm{NH}_{2}$; exchangeable with $\mathrm{D}_{2} \mathrm{O}$ ), 6.52 (d, $2 \mathrm{H}, o-\mathrm{NH}_{2}$; $J=8.4 \mathrm{~Hz}$ ), 6.56 (s, 1H, H-furan), 7.16-7.19 (m, 2H,o-tolyl), 7.23 (d, 1H, o-tolyl; $J=6.9 \mathrm{~Hz}$ ), 7.37 (d, 1H, o-tolyl; $J=7.7 \mathrm{~Hz}), 7.6(\mathrm{~s}, 1 \mathrm{H}, \mathrm{CH}=\mathrm{N}), 7.69$ (d, 2H, $\left.m-\mathrm{NH}_{2} ; J=8.4 \mathrm{~Hz}\right), 9.71$ (s, $1 \mathrm{H}, \mathrm{NH}$; exchangeable with $\left.\mathrm{D}_{2} \mathrm{O}\right), 10.27\left(\mathrm{~s}, 1 \mathrm{H}, \mathrm{NH}\right.$; exchangeable with $\mathrm{D}_{2} \mathrm{O}$ ); Anal. Calcd for $\mathrm{C}_{23} \mathrm{H}_{24} \mathrm{~N}_{6} \mathrm{O}_{2} \mathrm{~S}$ (448.54): C, 61.59; H, 5.39; N, 18.74 Found: C, 61.44; H, 5.28; N, 18.66.

\subsection{Reactions of Thiosemicarbazones 14-19 with Acetic Anhydride}

A mixture of 14-19 $(0.01 \mathrm{~mol})$, acetic anhydride $(10 \mathrm{~mL}, 0.1 \mathrm{~mol})$ was gently refluxed for $2 \mathrm{~h}$. The hot solution was poured onto ice water $(10 \mathrm{~mL})$ and the dihydro-1,3,4-thiadiazole which separated was filtered off, washed several times by water and dried.

5-(5-Acetamido-4-acetyl-4,5-dihydro-1,3,4-thiadiazol-2-yl)-2-methyl-N'-(1-phenylethylidene)furan-3carbohydrazide (20). Yield 65\%. Recrystallized from ethanol as white needles; m.p. $220-221{ }^{\circ} \mathrm{C}$; $\mathrm{R}_{\mathrm{f}}$ : $0.65\left(\mathrm{CHCl}_{3} / \mathrm{MeOH}, 25: 1, \mathrm{v} / \mathrm{v}\right)$; IR (KBr): $1597(\mathrm{C}=\mathrm{N}), 1698(\mathrm{CONH}), 1715$ (CO-acetyl), 3135, $3219 \mathrm{~cm}^{-1}$ $(2 \mathrm{NH}) ;{ }^{1} \mathrm{H}-\mathrm{NMR}\left(\mathrm{DMSO}-d_{6}\right) \delta: 1.98\left(\mathrm{~s}, 3 \mathrm{H}, \mathrm{CH}_{3} \mathrm{C}=\mathrm{N}\right), 2.16(\mathrm{~s}, 6 \mathrm{H}, 2 \mathrm{~N}-\mathrm{Ac}), 2.25\left(\mathrm{~s}, 3 \mathrm{H}, \mathrm{CH}_{3}\right.$-furan), 7.21 (d, 1H, H-furan), 7.23 (s, 1H, H-thiadiazolyl), 7.30-7.32 (m, 5H, Ar-H), 9.23 (bs, 1H, NH; exchangeable with $\left.\mathrm{D}_{2} \mathrm{O}\right), 11.60\left(\mathrm{~s}, 1 \mathrm{H}, \mathrm{NH}\right.$; exchangeable with $\left.\mathrm{D}_{2} \mathrm{O}\right) ; \mathrm{MS}: m / z(\%), 59(6.70), 77$ (29.21), 78 (9.60), 91 (5.22), 92 (5.37), 103 (14.25), 104 (13.30), 116 (9.86), 117 (6.74), 118 (25.31), 119 (7.62), 120 (4.38), 121 (12.26), 133 (15.23), 134 (5.36), 150 (5.09), 158 (12.76), 178 (32.05), 220 (100), 221 (13.32), 222 (5.74), 235 (13.82), 262 (13.77), 277 (28.09), 427 (5.09, $\mathrm{M}^{+}$); Anal. Calcd for $\mathrm{C}_{20} \mathrm{H}_{21} \mathrm{~N}_{5} \mathrm{O}_{4} \mathrm{~S}$ (427.48): C, 56.19; H, 4.95; N, 16.38 Found: C, 55.96; H, 4.81; N, 16.14.

4-(1-(5-(5-(N-Acetylacetamido)-4-acetyl-4,5-dihydro-1,3,4-thiadiazol-2-yl)-2-methylfuran-3-carboyl imino)ethyl)phenylacetate (21). Yield 42\%. Recrystallized from ethanol as white crystals; m.p. 123-124 ${ }^{\circ} \mathrm{C}$; $\mathrm{R}_{\mathrm{f}}: 0.41$ (n-hexane/EtOAc, 7:1, v/v); IR (KBr): $1593(\mathrm{C}=\mathrm{N}), 1671(\mathrm{CONH}), 1680$ (CO-acetyl), $3237 \mathrm{~cm}^{-1}(\mathrm{NH}) ;{ }^{1} \mathrm{H}-\mathrm{NMR}$ (DMSO-d 6 ) $\delta: 1.24\left(\mathrm{~s}, 3 \mathrm{H}, \mathrm{CH}_{3} \mathrm{C}=\mathrm{N}\right), 2.14$ (s, 3H, N-Ac), 2.21 (s, 3H, O-Ac), 2.27 (s, 3H, $\mathrm{CH}_{3}$-furan), 2.34 (s, 6H, N-(Ac) $)$ ), 6.69 (s, 1H, H-furan), 7.06 (s, 1H, 
H-thiadiazolyl), 7.23-7.26 (m, 4H, Ar-H), 8.22 (s, 1H, NH; exchangeable with $\mathrm{D}_{2} \mathrm{O}$ ); Anal. Calcd for $\mathrm{C}_{24} \mathrm{H}_{25} \mathrm{~N}_{5} \mathrm{O}_{7} \mathrm{~S}$ (527.55): C, 54.64; H, 4.78; N, 13.28 Found: C, 54.52; H, 4.64; N, 13.17.

5-(5-(N-Acetylacetamido)-4-acetyl-4,5-dihydro-1,3,4-thiadiazol-2-yl)-N'-(1-(4-acetamidophenyl)ethylidene)-2-methylfuran-3-carbohydrazide (22). Yield 73\%. Recrystallized from ethanol as buff needles; m.p. $152-135^{\circ} \mathrm{C}$; R $\mathrm{R}_{\mathrm{f}} 0.47$ (n-hexane/EtOAc, 7:1, v/v); IR (KBr): $1610(\mathrm{C}=\mathrm{N}), 1651(\mathrm{CONH})$, 1695 (CO-acetyl), 3232, $3345 \mathrm{~cm}^{-1}(2 \mathrm{NH}) ;{ }^{1} \mathrm{H}-\mathrm{NMR}\left[\left(\mathrm{CH}_{3}\right)_{2} \mathrm{CO}-d_{6}\right] \delta: 2.03\left(\mathrm{~s}, 3 \mathrm{H}, \mathrm{CH}_{3} \mathrm{CN}\right), 2.08$ (s, $3 \mathrm{H}, \mathrm{N}-\mathrm{Ac}$ ), 2.11 (s, 3H, N-Ac), 2.29 (s, 3H, $\mathrm{CH}_{3}$-furan), 2.48(s, 6H, N(Ac) $)_{2}$ ), 7.22 (d, 1H, H-furan), 7.30 (d, 2H, $m$-NAc; $J=8.4 \mathrm{~Hz}$ ), 7.44 (s, 1H, H-thiadiazolyl), 7.56 (d, 2H, o-NAc; $J=8.4 \mathrm{~Hz}$ ), 9.23 (s, 1H, NH; exchangeable with $\left.\mathrm{D}_{2} \mathrm{O}\right), 10.50$ (bs, $1 \mathrm{H}, \mathrm{NH}-\mathrm{Ac}$; exchangeable with $\left.\mathrm{D}_{2} \mathrm{O}\right)$; $\mathrm{MS}: \mathrm{m} / z(\%)$, 56 (31.73), 57 (20.17), 59 (30,76), 60 (21.91), 63 (7.39), 67 (12.24), 74 (60.64), 80 (5.05) 81 (8.21), 104 (7.24), 105 (5.83), 111 (7.53), 114 (16.47), 115 (100), 134 (13.51), 135 (12.88), 144 (6.56), 146 (4.86), 157 (15.11), 168 (5.88), 172 (5.88), 182 (5.73), 203 (6.66), 215 (7.19), 222 (5.00), 223 (5.49), 251 (9.33), 255 (8.41), 257 (4.71), 277 (17.15), 282 (5.78), 286 (5.34), 295 (5.49), 319 (5.78), 526 (5.34, $\mathrm{M}^{+}$); Anal. Calcd for $\mathrm{C}_{24} \mathrm{H}_{26} \mathrm{~N}_{6} \mathrm{O}_{6} \mathrm{~S}$ (526.56): C, 54.74; H, 4.98; N, 15.96 Found: C, 54.49; H, $4.81 ; \mathrm{N}, 15.70$.

5-(5-(N-o-Tolylacetamido)-4-acetyl-4,5-dihydro-1,3,4-thiadiazol-2-yl)-N-acetyl-2-methyl-N'-(1-phenyl ethylidene)furan-3-carbohydrazide (23). Yield 59\%. Recrystallized from ethanol as white needles; m.p. $107-108{ }^{\circ} \mathrm{C}$; $\mathrm{R}_{\mathrm{f}}: 0.87\left(\mathrm{CHCl}_{3} / \mathrm{MeOH}, 25: 1\right.$, v/v); IR (KBr): $1594(\mathrm{C}=\mathrm{N}), 1682 \mathrm{~cm}^{-1}$ (CO-acetyl); ${ }^{1} \mathrm{H}-\mathrm{NMR}\left(\mathrm{CHCl}_{3}-d\right) \delta: 1.88(\mathrm{~s}, 6 \mathrm{H}, 2 \mathrm{~N}-\mathrm{Ac}), 2.20\left(\mathrm{~s}, 3 \mathrm{H}, \mathrm{CH}_{3} \mathrm{CN}\right), 2.28$ (s, 3H, $\mathrm{CH}_{3}$-furan), 2.35 (s, $3 \mathrm{H}, \mathrm{CH}_{3}$-o-tolyl), 2.38 (s, 3H, N-Ac), 6.81 (s, 1H, H- furan), 7.17 (s, 1H, H-thiadiazolyl), 7.21-7.25 (m, 3H, Ar-H), 7.31-7.40 (m, 6H, Ar-H); MS: m/z (\%),65 (7.53), 77 (27.45), 78 (9.15), 91 (17.54), 103 (13.66), 104 (18.10), 107 (23.20), 118 (28.32), 121 (19.42), 133 (18.36), 149 (7.33), 150 (7.61), 161 (13.18), 206 (11.26), 221 (0.67), 248 (14.33), 250 (9.44), 268 (45.35), 310 (100), 311 (20.21), 325 (17.89), 367 (47.84), 559(13.18, $\mathrm{M}^{+}$); Anal. Calcd for $\mathrm{C}_{29} \mathrm{H}_{29} \mathrm{~N}_{5} \mathrm{O}_{5} \mathrm{~S}$ (559.64): C, 62.24; H, 5.22; N, 12.51 Found: C, 62.50; H, 4.99; N, 12.38 .

4-(1-(5-(5-(N-o-Tolylacetamido)-4-acetyl-4,5-dihydro-1,3,4-thiadiazol-2-yl)-2-methylfuran-3-carboylimino)ethyl)phenyl acetate (24). Yield 40\%. Recrystallized from ethanol as buff needles; m.p. $119-120{ }^{\circ} \mathrm{C}$; $\mathrm{R}_{\mathrm{f}}: 0.28\left(\mathrm{CHCl}_{3} / \mathrm{MeOH}, 25: 1, \mathrm{v} / \mathrm{v}\right)$; IR $(\mathrm{KBr}): 1560(\mathrm{C}=\mathrm{N}), 1652(\mathrm{CONH}), 1699$ (CO-acetyl), 3233 $\mathrm{cm}^{-1}(\mathrm{NH}) ;{ }^{1} \mathrm{H}-\mathrm{NMR}\left(\mathrm{DMSO}-d_{6}\right) \delta: 1.71\left(\mathrm{~s}, 3 \mathrm{H}, \mathrm{CH}_{3} \mathrm{CN}\right), 1.79(\mathrm{~s}, 6 \mathrm{H}, \mathrm{N}-\mathrm{Ac}, \mathrm{O}-\mathrm{Ac}), 1.87(\mathrm{~s}, 3 \mathrm{H}$, $\mathrm{CH}_{3}$-furan), 1.93 (s, 3H, $\mathrm{CH}_{3}-\mathrm{O}$-tolyl), 6.72 (s, 1H, H-furan), 6.78 (d, 2H,o-OAc; $J=8.4 \mathrm{~Hz}$ ), 7.13-7.20 (m, 2H, o-tolyl-H), 7.21 (d, 1H, o-tolyl-H; $J=6.9 \mathrm{~Hz}$ ), 7.29 (d, 1H, o-tolyl-H; $J=7.7 \mathrm{~Hz}$ ), 7.70 (d, $2 \mathrm{H}, m$-OAc; $J=8.4 \mathrm{~Hz}), 7.81$ (s, $1 \mathrm{H}, \mathrm{H}$-thiadiazolyl), 8.85 (s, 1H, NH; exchangeable with $\mathrm{D}_{2} \mathrm{O}$ ), 9.69 (s, $1 \mathrm{H}, \mathrm{NH}$; exchangeable with $\mathrm{D}_{2} \mathrm{O}$ ). Anal. Calcd for $\mathrm{C}_{29} \mathrm{H}_{29} \mathrm{~N}_{5} \mathrm{O}_{6} \mathrm{~S}(533.6)$ : C, 60.51; H, 5.08; N, 12.17 Found: C, 60.37; H, 4.99; N, 12.05.

5-(5-(N-o-Tolylacetamido)-4-acetyl-4,5-dihydro-1,3,4-thiadiazol-2-yl)-N'-(1-(4-(N-acetylacetamido) phenyl)ethylidene)-2-methylfuran-3-carbohydrazide (25). Yield 69\%. Recrystallized from ethanol as yellow needles; m.p. $114-115{ }^{\circ} \mathrm{C}$; $\mathrm{R}_{\mathrm{f}}$ : $0.65\left(\mathrm{CHCl}_{3} / \mathrm{MeOH}, 25: 1, \mathrm{v} / \mathrm{v}\right)$; IR $(\mathrm{KBr}): 1598(\mathrm{C}=\mathrm{N}), 1647$ (CONH), 1677 (CO-acetyl), $3377 \mathrm{~cm}^{-1}(\mathrm{NH})$; Anal. Calcd for $\mathrm{C}_{31} \mathrm{H}_{32} \mathrm{~N}_{6} \mathrm{O}_{6} \mathrm{~S}$ (616.69): C, 60.38; $\mathrm{H}$, 5.23; N, 13.63 Found: C, 60.26; H, 5.31; N, 13.74 . 


\subsection{Reactions of 5-Formyl-2-methyl-N'-(1-arylethylidene)furan-3-carbohydrazides 10-12 with p-tosylhydrazine}

A solution of 5-formyl-2-methyl-N'-(1-arylethylidene)furan-3-carbohydrazide 10-12 (0.001 mol) in ethanol $(30 \mathrm{~mL})$ containing acetic acid $(0.01 \mathrm{~mL})$ was treated with $p$-tosylhydrazine $(0.196 \mathrm{~g}, 0.001 \mathrm{~mol})$. The mixture was refluxed for 3-4 h. After cooling, the product which separated out was filtered off, washed with little ethanol and dried.

1-((4-(1-Phenylethylideneaminocarbamoyl)furan-2-yl)-2-p-tosylhydrazine methylene (26). Yield 57\%. Recrystallized from ethanol as white crystals; m.p. $115-116{ }^{\circ} \mathrm{C} ; \mathrm{R}_{\mathrm{f}}: 0.88\left(\mathrm{CHCl}_{3} / \mathrm{MeOH}, 20: 1\right.$, v/v); IR $(\mathrm{KBr}): 1163,1335\left(\mathrm{SO}_{2}\right), 1599(\mathrm{C}=\mathrm{N}), 1659(\mathrm{CONH}), 3223 \mathrm{~cm}^{-1}(\mathrm{NH}) ;{ }^{1} \mathrm{H}-\mathrm{NMR}\left[\left(\mathrm{CH}_{3}\right)_{2} \mathrm{CO}-d_{6}\right] \delta$ : 2.21 (s, 3H, $\mathrm{CH}_{3} \mathrm{CN}$ ), 2.30 (s, 3H, $\mathrm{CH}_{3}$-furan), 2.36 (s, 3H, $\mathrm{CH}_{3}$-tolyl), 7.32-7.36 (m, 3H, Ph), 7.41-7.43 (m, 2H, $m-\mathrm{H}$ of $\mathrm{Ph}), 7.68-7.69$ (m, 2H, $o$-H of $p$-tolyl), 7.83 (s,1H, H-furan), 7.85 (s, 1H, $\mathrm{CH}=\mathrm{N}), 7.93-7.95\left(\mathrm{~m}, 2 \mathrm{H}, m-\mathrm{H}\right.$ of $p$-tolyl), $9.36\left(\mathrm{~s}, 2 \mathrm{H}, 2 \mathrm{NH}\right.$; exchangeable with $\left.\mathrm{D}_{2} \mathrm{O}\right)$; $\mathrm{MS}: \mathrm{m} / z(\%)$, 65 (40.65), 77 (30.59), 78 (25.23), 91 (33.65), 92 (90.68), 104 (100), 118 (20.56), 132 (30.51), 133 (85.78), 134 (10.56), 140 (5.36), 288 (8.96), 438 (10.56, $\mathrm{M}^{+}$). Anal. Calcd for $\mathrm{C}_{22} \mathrm{H}_{22} \mathrm{~N}_{4} \mathrm{O}_{4} \mathrm{~S}$ (438.5): C, 60.26; H, 5.06; N, 12.78 Found: C, 60.05; H, 4.93; N, 12.53.

1-((4-(1-(4-Hydroxyphenylethylideneaminocarbamoyl)furan-2-yl)methylene-2-p-tosylhydrazine (27). Yield $42 \%$. Recrystallized from ethanol as orange crystals; m.p. $269-270{ }^{\circ} \mathrm{C}, \mathrm{R}_{\mathrm{f}}: 0.92\left(\mathrm{CHCl}_{3} / \mathrm{MeOH}\right.$, 15:1, v/v); IR (KBr): 1164, $1337\left(\mathrm{SO}_{2}\right), 1598(\mathrm{C}=\mathrm{N}), 1658(\mathrm{CONH}), 3225,3447 \mathrm{~cm}^{-1}(\mathrm{NH}, \mathrm{OH}) ;{ }^{1} \mathrm{H}-\mathrm{NMR}$ $\left(\mathrm{DMSO}-d_{6}\right) \delta: 2.14\left(\mathrm{~s}, 3 \mathrm{H}, \mathrm{CH}_{3} \mathrm{CN}\right), 2.32\left(\mathrm{~s}, 6 \mathrm{H}, \mathrm{CH}_{3}\right.$-furan, $\mathrm{CH}_{3}$ of $p$-tolyl), 4.99 (bs, $1 \mathrm{H}, \mathrm{OH}$; exchangeable with $\left.\mathrm{D}_{2} \mathrm{O}\right), 6.70(\mathrm{~d}, 1 \mathrm{H}, \mathrm{H}$-furan; $J=8.5 \mathrm{~Hz}), 7.37(\mathrm{~d}, 2 \mathrm{H}, o-\mathrm{H}$ of $p$-tolyl; $J=7.7 \mathrm{~Hz}$ ), $7.33\left(\mathrm{t}, 2 \mathrm{H}, o-\mathrm{OH} ; J_{1,2}=3.1, J_{1,3}=5.4 \mathrm{~Hz}\right), 7.58\left(\mathrm{t}, 2 \mathrm{H}, m-\mathrm{OH} ; J_{1,2}=3.1, J_{1,3}=5.4 \mathrm{~Hz}\right), 7.69(\mathrm{~s}, 1 \mathrm{H}$, $\mathrm{CH}=\mathrm{N}), 7.78(\mathrm{~d}, 2 \mathrm{H}, m-\mathrm{H}$ of $p$-tolyl; $J=7.7 \mathrm{~Hz}), 9.71\left(\mathrm{~s}, \mathrm{H}, \mathrm{NH}\right.$; exchangeable with $\left.\mathrm{D}_{2} \mathrm{O}\right), 10.47(\mathrm{~s}, \mathrm{H}$, $\mathrm{NH}$; exchangeable with $\left.\mathrm{D}_{2} \mathrm{O}\right)$; MS: $m / z(\%), 59$ (7.33), 81 (4.23), 108 (19.85), 119 (11,00), 120 (21.47), 121 (16.05), 149 (31.06), 150 (7.52), 155 (6.25), 156 (6.25), 158 (12.69), 178 (27.39), 220 (100), 221 (13.15), 235 (13.28), 262 (15.08), 277 (29.17), 278 (6.49), 454 (15.08, $\mathrm{M}^{+}$). Anal. Calcd for $\mathrm{C}_{22} \mathrm{H}_{22} \mathrm{~N}_{4} \mathrm{O}_{5} \mathrm{~S}$ (454.5): C, 58.14; H, 4.88; N, 12.33 Found: C, 57.96; H, 4.75; N, 12.22.

1-((4-(1-(4-Aminophenylethylideneaminocarbamoyl)furan-2-yl)methylene-2-p-tosylhydrazine (28).

Yield $83 \%$. Recrystallized from ethanol as yellow crystals, m.p. $160-162{ }^{\circ} \mathrm{C} ; \mathrm{R}_{\mathrm{f}}: 0.61\left(\mathrm{CHCl}_{3} / \mathrm{MeOH}\right.$, 25:1, v/v); IR (KBr): 1182, $1363\left(\mathrm{SO}_{2}\right), 1589(\mathrm{C}=\mathrm{N}), 1661(\mathrm{CONH}), 3238,3391,3429 \mathrm{~cm}^{-1}(\mathrm{NH}$, $\mathrm{NH}_{2}$ ). Anal. Calcd for $\mathrm{C}_{22} \mathrm{H}_{23} \mathrm{~N}_{5} \mathrm{O}_{4} \mathrm{~S}$ (453.51): C, 58.26; H, 5.11; N, 15.44 Found: C, 58.19; H, 5.02; N, 15.40 .

\subsection{Reactions of 26-28 with Acetic Anhydride}

A mixture of 1-((4-(1-arylethylidene aminocarbamoyl)furan-2-yl)methylene-2- $p$-tosylhydrazine 26-28 $(0.0005 \mathrm{~mol})$, acetic anhydride $(20 \mathrm{~mL})$ was gently refluxed for $20 \mathrm{~min}$. The hot solution was poured onto ice water $(10 \mathrm{~mL})$, the 1,2,3,4-oxathiadiazole product which separated was filtered off, washed several times by water and dried. 
2-Methyl-N'-(1-phenylethylidene)-5-(2-p-tolyl-1,2,3,4-oxathiadiazol-5-yl)furan-3-carbohydrazide (29). Yield 54\%. Recrystallized from ethanol as buff needles; m.p. $174-175{ }^{\circ} \mathrm{C} ; \mathrm{R}_{\mathrm{f}}: 0.83\left(\mathrm{CHCl}_{3} / \mathrm{MeOH}\right.$, 25:1, v/v); IR (KBr): $1598(\mathrm{C}=\mathrm{N}), 1645(\mathrm{CONH}), 1710$ (CO-acetyl), $3260 \mathrm{~cm}^{-1}(\mathrm{NH})$; ${ }^{1} \mathrm{H}-\mathrm{NMR}$ $\left(\mathrm{CHCl}_{3}-d\right) \delta: 2.05$ (s, 3H, $\left.\mathrm{CH}_{3} \mathrm{CN}\right), 2.40$ (s, 3H, $\mathrm{CH}_{3}$-furan), 2.43 (s, 3H, $\mathrm{CH}_{3}$ p-tolyl), 7.26-7.33 (m, 4H, $p$-tolyl), 7.40 (s,1H, H-furan), 7.44-7.48 (m, 1H, $p$-H of $\mathrm{Ph}), 7.76$ (d, 2H, $m-\mathrm{H}$ of $\mathrm{Ph} ; J=8.4 \mathrm{~Hz}$ ), $7.94(\mathrm{~d}, 2 \mathrm{H}, o-\mathrm{H}$ of $\mathrm{Ph} ; J=8.4 \mathrm{~Hz}), 8.19$ (bs, $1 \mathrm{H}, \mathrm{NH}$; exchangeable with $\left.\mathrm{D}_{2} \mathrm{O}\right)$; MS: $m / z(\%), 51$ (15.47), 65 (24.48), 77 (47.41), 91 (55.26), 103 (96.40), 104 (22.97), 105 (21.46), 119 (12.93), 133 (100), 134 (9.78), 139 (17.48), 160 (78.93), 175 (55.76), $420\left(9.77, \mathrm{M}^{+}\right.$); Anal. Calcd for $\mathrm{C}_{22} \mathrm{H}_{20} \mathrm{~N}_{4} \mathrm{O}_{3} \mathrm{~S}$ (420.48): C, 62.84; H, 4.79; N, 13.32 Found: C, 62.95; H, 4.59; N, 13.19.

4-(1-(2-Methyl-5-(2-p-tolyl-1,2,3,4-oxathiadiazol-5-yl)furan-3-carboylimino)ethyl) phenyl acetate (30). Yield $42 \%$. Recrystallized from ethanol as yellow needles; m.p. $104-105{ }^{\circ} \mathrm{C} ; \mathrm{R}_{\mathrm{f}}: 0.26\left(\mathrm{CHCl}_{3} / \mathrm{MeOH}\right.$, 25:1, v/v); IR (KBr): $1597(\mathrm{C}=\mathrm{N}), 1643(\mathrm{CONH}), 1713$ (CO-acetyl), $3368 \mathrm{~cm}^{-1}(\mathrm{NH})$; MS: $m / z(\%), 51$ (15.47), 65 (91.77), 77 (78.93), 91 (71.33), 103 (9.40), 105 (23.11), 107 (100), 119 (63.07), 133 (60.24), 134 (65.21), 149 (36.02), 150 (23.07), 160 (9.03), 164 (45.44), 175 (12.24), 253 (96.40), 261 (0.21), 267 (11.78), 268 (45.26), 283 (23.05), 296 (12.45, 478 (24.48, $\left.\mathrm{M}^{+}\right)$; Anal. Calcd for $\mathrm{C}_{24} \mathrm{H}_{22} \mathrm{~N}_{4} \mathrm{O}_{5} \mathrm{~S}$ (478.52): C, 60.24; H, 4.63; N, 11.71 Found: $\mathrm{C}, 60.07 ; \mathrm{H}, 4.54 ; \mathrm{N}, 11.60$.

N'-(1-(4-Acetamidophenyl)ethylidene)-2-methyl-5-(2-p-tolyl-1,2,3,4-oxathiadiazol-5-yl)furan-3-carbohydrazide (31). Yield 32\%. Recrystallized from ethanol as yellow needles; m.p. $111-112{ }^{\circ} \mathrm{C}$; $\mathrm{R}_{\mathrm{f}}: 0.58$ $\left(\mathrm{CHCl}_{3} / \mathrm{MeOH}, 25: 1, \mathrm{v} / \mathrm{v}\right)$; IR (KBr): $1599(\mathrm{C}=\mathrm{N}), 1645(\mathrm{CONH}), 1710$ (CO-acetyl), $3390(\mathrm{NH}) ;{ }^{1} \mathrm{H}-\mathrm{NMR}$ $\left[\left(\mathrm{CH}_{3}\right)_{2} \mathrm{CO}-d_{6}\right] \delta: 2.09$ (s, 6H, $\mathrm{CH}_{3} \mathrm{CN}, \mathrm{CH}_{3} \mathrm{CO}$ ), 2.49 (s, 3H, $\mathrm{CH}_{3}$-furan), 2.49 (s, 3H, $\mathrm{CH}_{3} p$-tolyl), 7.64 (s, 1H, H-furan), 7.73 (d, 4H, p-tolyl; $J=9.2 \mathrm{~Hz}$ ), 7.90 (d, 4H, Ar-H; $J=8.4 \mathrm{~Hz}$ ), 9.57 (bs, 2H, 2NH; exchangeable with $\mathrm{D}_{2} \mathrm{O}$ ); Anal. Calcd for $\mathrm{C}_{24} \mathrm{H}_{23} \mathrm{~N}_{5} \mathrm{O}_{4} \mathrm{~S}$ (477.54): C, 60.36; H, 4.85; N, 14.67 Found: C, 60.19; H, 4.71; N, 14.63.

\section{Conclusions}

Some new $C$-nucleoside derivatives, thiadiazole and oxathiadiazole derivatives have been prepared as well as their physical properties and biological effect on the enzyme tyrosinase studied.

\section{Acknowledgments}

We would like to thank Mkhyoon, M. of Inorganic Chemistry, Faculty of Science, Alexandria University for his help in theoretical calculations.

\section{References}

1. Dogan, H.N.; Duran, A.; Rollas, S.; Sener, G.; Uysal, M.K.; Gulen, D. Synthesis of new 2,5-disubstituted-1,3,4-thiadi azoles and preliminary evaluation of anticonvulsant and antimicrobial activities. Bioorg. Med. Chem. Lett. 2002, 10, 2893-2898.

2. Mamolo, M.G.; Falagiani, V.; Zampieri, D.; Vio, L.; Banfi, E.; Scialino, G. Synthesis and antimycobacterial activity of (3,4-diaryl-3H-thiazol-2-ylidene)-hydrazide derivatives. Farmaco 2003, 58, 631-637. 
3. Foroumadi, A.; Kiani, Z.; Soltani, F. Antituberculosis agents VIII-Synthesis and in vitro antimycobacterial activity of alkyl $\alpha$-[5-(5-nitro-2-thienyl)-1,3,4-thiadiazole-2-ylthio] acetates. Farmaco 2003, 58, 1073-1076.

4. Oruc, E.E.; Rollas, S.; Kandemirli, F.; Shvets, N.; Dimoglo, A.S. 1,3,4-thiadiazole derivatives. synthesis, structure elucidation, and structure-antituberculosis activity relationship investigation. J. Med. Chem. 2004, 47, 6760-6767.

5. Stillings, M.R.; Welbourn, A.P.; Walter, D.S. Substituted 1,3,4-thiadiazoles with anticonvulsant activity. 2. Aminoalkyl derivatives. J. Med. Chem. 1986, 29, 2280-2284.

6. Chapleo, C.B.; Myers, P.L.; Smith, A.C.; Tulloch, I.F.; Walter, D.S. Substituted 1,3,4-thiadiazole with anticonvulsant activity. J. Med. Chem. 1987, 30, 951-954.

7. Chimirri, A.; Grasso, S.; Monforte, A.M.; Zappala, M. Synthesis and anticonvulsant properties of 3-(1,3,4-thiadiazol-2-yl)thiazolidin-4-ones. Farmaco 1991, 46, 935-943.

8. Clerici, F.; Pocar, D.; Maddalena, G.; Loche, A.; Perlini, V.; Brufani, M. Synthesis of 2-amino-5sulfanyl-1,3,4-thiadiazole derivatives and evaluation of their antidepressant and anxiolytic activity. J. Med. Chem. 2001, 44, 931-936.

9. Vergne, F.; Bernardelli, P.; Lorthiois, E.; Pham, N.; Proust, E.; Oliveira, C.; Mafroud, A.; Royer, F.; Wrig-glesworth, R.; Schellhaas, J.K.; et al. Discovery of thiadiazoles as a novel structural class of potent and selective PDE7 inhibitors. Part 1. design, synthesis and structureactivity relationship studies. Bioorg. Med. Chem. Lett. 2004, 14, 4607-4613.

10. Song, Y.; Connor, D.T.; Sercel, A.D.; Sorenson, R.J.; Doubleday, R. Synthesis, structure activity relationships, and in vivo evaluations of substituted di-tert-butylphenols as a novel class of potent, selective, and orally active cyclooxygenase-2 inhibitors. 2. 1,3,4- and 1,2,4-thiadiazole series. J. Med. Chem. 1999, 42, 1161-1169.

11. Labanauskas, L.; Kalacs, V.; Gaidelis, P.; Brukstus, A.; Dauksas, V. Synthesis of 3-(3,4dimethoxy phenyl)-1H-1,2,4-triazole-5-thiol and 2-amino) 3,4-dimethoxy phenyl)-1,3,4thiadiazole derivatives exhibiting antiinflammatory Activity. Pharmazie 2001, 56, 617-619.

12. Sahin, G.; Palaska, E.; Kelicen, P.; Demirdamar, R.; Altinok, G.A. Synthesis of some new 3-acyl thiosemicarbazides, 1,3,4-oxadiazoles, 1,3,4-thiadiazoles and 1,2,4-triazole-1-thiones and their anti-inflammatory activities. Forsch Drug Res. 2001, 51, 478-484.

13. Palaska, E.; Sahin, G.; Kelicen, P.; Durlu, N.T.; Altinok, G. Synthesis and anti-inflammatory activity of 1-acylthiosemicarbazides, 1,3,4-oxadiazoles, 1,3,4-thiadiazoles and 1,2,4-triazole-3thiones. Il Farmaco 2002, 57, 101-107.

14. Amir, M.; Shikha, K. Synthesis and antiinflammatory, analgesic, ulcerogenic and lipid peroxidation activities of some new 2-[(2,6-dichloroanilino)phenyl]acetic acid derivatives. Eur. J. Med. Chem. 2004, 39, 535-545.

15. Mishra, L.; Singh, V.K.; Dubey, N.K.; Mishra, A.K. Synthesis and fungicidal activity of some 5membered heterocyclic derivatives containing benzimidazoles. Biosci. Biotechnol. Biochem. 1993, 57, 989-991.

16. Dogan, H.N.; Rollas, S.; Erdeniz, H. Synthesis, structure elucidation and antimicrobial activity of some 3-hydroxy-2- naphthoic acid hydrazide derivatives. Il Farmaco 1998, 53, 462-467.

17. Mamolo, M.G.; Vio, L.; Banfi, E. Synthesis and antimicrobial activity of some 2,5-disubstituted 1,3,4-thiadiazole derivatives. Il Farmaco 1996, 51, 71-74. 
18. Chufan, E.E.; Pedregosa, J.C.; Baldini, O.N.; Bruno-Blanch, L. Anticonvulsant activity of analogues of acetazolamide. Il Farmaco 1999, 54, 838-841.

19. Krutovskikh, G.N.; Rusanov, A.M.; Gornaeva, G.F.; Vartanyan, L.P.; Kolesova, M.B. Radioprotective action of thiadiazole derivatives. Pharm. Chem. J. (Engl. Transl.) 1977, 11, 484-488; Kim.-Farm. Zh. 1977, 11, 48-52.

20. Chou, J.Y.; Lai, S.Y.; Pan, S.L.; Jow, G.M.; Chern, J.W.; Guh, J.H. Investigation of anticancer mechanism of thiadiazole- based compound in human nonsmall cell lung cancer A549 cells. Biochem. Pharmacol. 2003, 66, 115-124.

21. Oleson, J.J.; Sloboda, A.; Troy, W.P.; Halliday, S.L.; Landes, M.J.; Angier, R.B.; Semb, J.; Cyr, K.; Williams, J.H. The carcinostatic activity of some 2-amino-1.3,4-thiadiazoles. J. Am. Chem. Soc. 1955, 77, 6713-6714.

22. El-Sadek, M.M.; Mostafa, M.A.; Abdel Rahman, M.M.; Zagzoug, N.B. Reactions of periodate oxidized methyl - 4,6-O-benzylidene- $\alpha$-D-glucopyranoside with hydrazines. Bratislava Symposia on Saccharides, Smollenice, Czechoslovakia 1984, 67.

23. El-Sadek, M.M.; Faidallah, H.M.; Hassan, S.Y. Structure and reactions of 3-benzoyl-2-methyl-5(D-arabino- tetrahydroxybutyl)pyrrole. Carbohydr. Res. 1990, 199, 248-254.

24. Hassan, S.Y.; Faidallah, H.M.; El Massry, A.; El Sadek, M.M. Synthesis and reactions of 5-(D-arabino-tetrahydroxybutyl)-3-(2,3-dihydro-1,3,4-oxadiazole-2-thion-5-yl)-2-methylfuran and 5-(D-arabino-tetrahydroxybutyl)-3-(2substituted amino-1,3,4-oxadiazol-5-yl)-2-methyl furan. Carbohydr. Res. 1997, 298, 123-126.

25. El-Sadek, M.M.; El Soccary, N.N. Synthesis of N-[1-acetyl-5-(d-arabino-tetraacetoxybutyl)-2methyl-3-pyrroyl]-N-phthalimidoacetamide. Carbohydr. Res. 1991, 222, 267-269.

26. El-Sadek, M.M.; Abdel-baky, S.A.; El Soccary, N.N. Synthesis and reactions of 2-methyl 5-(Darabino-tetrahydroxy butyl)-3-pyrrolecarbohydrazide. Carbohydr. Res. 1992, 223, 311-319.

27. El-Sadek, M.M.; Zagzoug, N.B.; El Soccary, N.N. Reactions of 2-methyl-5-(D-arabinotetrahydroxybutyl)-3-furoylhydrazine. Carbohydr. Res. 1993, 250, 323-326.

28. Barton, D.E.; Kwon, B.S.; Francke, U. Human tyrosinase gene, mapped to chromosome 11 $(\mathrm{q} 14 \rightarrow \mathrm{q} 21)$, defines second region of homology with mouse chromosome 7. Genomics 1988, 3, $17-24$.

29. Oetting, W.S.; Fryer, J.P.; Shriram, S.; King, R.A. Oculocutaneous albinism type 1: The last 100 years. Pigment Cell Res. 2003, 16, 307-311.

30. Potterf, S.B.; Hearing, V.J. Tyrosine transport into melanosomes is increased following stimulation of melanocyte differentiation. Biochem. Biophys. Res. Commun. 1998, 248, 795-800.

31. Borden, E.C. Melanoma: Biologically Targeted Therapeutics; Humana Press: New York, NY, USA, 2002.

32. Arias-Carrión, O.; Pöppel, E. Dopamine, learning, and reward-seeking behavior. Acta Neurobiol. Exp. 2007, 67, 481-488.

33. Seo, S.Y.; Sharma, V.K.; Sharma, N.J. Mushroom Tyrosinase: Recent Prospects. Agric. Food Chem. 2003, 51, 2837-2853.

34. Gonzalez, G. Reactions of mono saccharides with $\beta$-keto-esters. Adv. Carbohydr. Chem. 1956, 11, 97-143. 
35. El-Sadek, M.M.; Zagzoug, N.B. Synthesis and reactions of 2-methyl-5-(d-arabinotetrahydroxybutyl)-3-furoylhydra zine. Carbohydr. Res. 1991, 212, 261-265.

36. ElKhadem, H.; Horton, D.; Page, T.F. Structure of osotriazoles of the sugars. Conformational and configurational, correlations of the poly hydroxyl alkyl chain. J. Org. Chem. 1968, 33, 734-740.

37. Sallam, M.A.E.; El Nahas, H.M.; Abdel Megid, S.M.E. Studies on the conformation of polyhydroxyalkylpyrazolo(3,4-b)quinoxalines. J. Carbohydr. Chem. 1986, 5, 33-48.

38. Pavia, D.L.; Lampman, G.M.; Kriz, G.S. Introduction to Spectroscopy; Thomson Learning Inc.: Belmont, CA, USA, 2001; Chapter 6, pp. 318-320.

39. Yang, Z.; Robb, D.A. Tyrosinase activityin reversedmicelles. Biocatal. Biotransform. 2005, 23, 423-430.

40. Yang, Z.; Wu, F. Catalytic properties of tyrosinase from potato and edible Fungi. Biotechnology 2006, 5, 344-348.

Sample Availability: Samples of the compounds 1-31 are available from the authors.

(C) 2012 by the authors; licensee MDPI, Basel, Switzerland. This article is an open access article distributed under the terms and conditions of the Creative Commons Attribution license (http://creativecommons.org/licenses/by/3.0/). 